\title{
Ouroboros Wear Leveling for NVRAM Using Hierarchical Block Migration
}

\author{
QINGYUE LIU and PETER VARMAN, Rice University
}

\begin{abstract}
Emerging nonvolatile RAM (NVRAM) technologies have a limit on the number of writes that can be made to any cell, similar to the erasure limits in NAND Flash. This motivates the need for wear leveling techniques to distribute the writes evenly among the cells. Unlike NAND Flash, cells in NVRAM can be rewritten without the need for erasing the entire containing block, avoiding the issues of space reclamation and garbage collection, motivating alternate approaches to the problem. In this article, we propose a hierarchical wear-leveling model called Ouroboros wear leveling. Ouroboros uses a two-level strategy whereby frequent low-cost intraregion wear leveling at small granularity is combined with interregion wear leveling at a larger time interval and granularity. Ouroboros is a hybrid migration scheme that exploits correct demand predictions in making better wear-leveling decisions while using randomization to avoid wear-leveling attacks by deterministic access patterns. We also propose a way to optimize wear-leveling parameter settings to meet a target smoothness level under limited time and space overhead constraints for different memory architectures and trace characteristics. Several experiments are performed on synthetically generated memory traces with special characteristics, two block-level storage traces, and two memory-line-level memory traces. The results show that Ouroboros wear leveling can distribute writes smoothly across the whole NVRAM with no more than $0.2 \%$ space overhead and $0.52 \%$ time overhead for a $512 \mathrm{~GB}$ memory.
\end{abstract}

CCS Concepts: • Hardware $\rightarrow$ External storage;

Additional Key Words and Phrases: Wear leveling, NVRAM, lifetime endurance

\section{ACM Reference format:}

Qingyue Liu and Peter Varman. 2017. Ouroboros Wear Leveling for NVRAM Using Hierarchical Block Migration. ACM Trans. Storage 13, 4, Article 30 (November 2017), 31 pages.

https://doi.org/10.1145/3139530

\section{INTRODUCTION}

Emerging byte-addressable persistent memory technologies (simply referred to as NVRAM in the article) such as Phase Change Memory (PCM) [4, 18-20, 31], Ferroelectric RAM (FeRAM) [1, 28], Spin-Torque Transfer Magnetoresistive RAM (STT-MRAM) [8, 12, 17], Resistive RAM (RRAM) [21, 27], and the recently announced 3D-XPoint technology [13] have been proposed as possible replacements for traditional nonvolatile storage. These devices have several attractive features such as high density, byte addressability, lower static power consumption, better scalability, direct processor access, and better read and write latencies than traditional storage devices.

Despite many advantages, almost all of these new NVRAM technologies suffer from the problem of limited write endurance. Write endurance limits the number of writes that can be made to a

This work is supported by NSF Grant CCF 1439075 and the Huawei Innovation Research Program (HIRPO20150401). Authors' addresses: Q. Liu, Elec \& Comp Eng MS-366, Rice University, PO Box 1892; email: q19@rice.edu; P. Varman, Elec \& Comp Engineering, 2022 Duncan Hall, 6100 Main Street MS 380; email: pjv@rice.edu.

Permission to make digital or hard copies of all or part of this work for personal or classroom use is granted without fee provided that copies are not made or distributed for profit or commercial advantage and that copies bear this notice and the full citation on the first page. Copyrights for components of this work owned by others than ACM must be honored. Abstracting with credit is permitted. To copy otherwise, or republish, to post on servers or to redistribute to lists, requires prior specific permission and/or a fee. Request permissions from permissions@acm.org.

(c) 2017 ACM 1553-3077/2017/11-ART30 $\$ 15.00$

https://doi.org/10.1145/3139530

ACM Transactions on Storage, Vol. 13, No. 4, Article 30. Publication date: November 2017. 
memory cell before it becomes unreliable. Compared with DRAM, which has a write endurance of more than $10^{16}$ writes per cell, PCM and RRAM are limited to $10^{7} \sim 10^{8}$ and $10^{5}$ writes per cell, respectively [23, 34], comparable to that reported for NAND Flash [29]. Since in reality writes are nonuniformly distributed among different memory cells, this nonuniformity in writes can result in a $20 \times$ lower lifetime than the ideal situation when writes are uniformly distributed across the whole memory [26].

Wear-leveling technology, which migrates heavily written memory regions to areas with lighter usage, is used to mitigate the write endurance problem. Wear leveling is not a new concept, nor is it specific to NVRAM. It was proposed around 30 years ago mainly for solving the wearout problem for NAND Flash $[2,5]$. While many of the wear-leveling approaches developed for NAND Flash can also be employed for NVRAM, specific technology characteristics such as in-place writing, word-line access granularity, and differences in read/write speed make direct use of these solutions undesirable. This has motivated the investigation of new wear-leveling approaches that take advantage of the special characteristics of NVRAM to design better solutions to the wear-out problem with lower overheads.

A number of NVRAM wear-leveling methods that remap frequently written memory lines to those with fewer writes have been proposed in the last decade $[6,7,11,14,15,22,24,26,30,32$, 35]. These solutions are of two types: those using restricted algebraic mappings to determine the target address of a relocated memory line and those using associative mappings maintained in a lookup mapping table.

For the first category [22, 26, 30,32], no address mapping table is needed. To permit computation of the current address of a memory line, migrations follow deterministic, easily computable mapping rules. Start-Gap [26] wear leveling is a representative method in this category. All memory lines within a region are rotated periodically using a simple circular shift of the memory lines; the shift is done incrementally with one line in a region being migrated to an adjacent spare (empty) line (called the GapLine) at any step. Memory line addresses can be tracked by two pointers START and GAP, which hold the current locations of the first memory line and the GapLine, respectively, in each region of the memory.

The size of the memory region determines the rotation period of the GapLine. If the period is too long, it is possible that some memory lines wear out before relocation. Therefore, in [26], the whole memory space is divided into multiple regions and wear leveling is done independently within each region. However, this also prevents wear leveling from exploiting uneven wear across different regions. Other wear-leveling methods in this category such as Curling-PCM [22, 30] slide a contiguous set of active blocks across the memory. This approach requires the hot variables to be placed in a contiguous memory region by the compiler and has been advocated for applicationspecific embedded system applications.

For the second category of wear-leveling methods, an additional table is used to store a full associative mapping from logical blocks to physical frames. Segment swapping [35] and PCMaware swap [9] are representative techniques that periodically swap the contents of a heavily used frame with a target frame. Segment swapping [35] chooses the frame with the lowest usage as the target frame, while in PCM-aware swap [9], the target frame is a randomly chosen frame. We refer to the first method as Deterministic Usage-based Segment Swap (DUSS) and the second method as Randomized Usage-based Segment Swap (RUSS) in this article. In Section 2, we will use microexperiments to demonstrate the drawbacks of both DUSS and RUSS. We will show that DUSS is vulnerable to severely uneven write distributions for some simple write patterns. Both targeted attacks and simple access patterns like repeated data writes to alternating buffers can result in hot spots to a few physical frames and cause them to wear out quickly despite the use of DUSS wear 
leveling. We will also show that RUSS fails to exploit favorable data migrations that could result in better write distributions across NVRAM.

In this article, we propose a novel hierarchical wear-leveling method called Ouroboros wear leveling suitable for NVRAM. Ouroboros uses a two-level solution. The first level uses local wear leveling within localized regions called frames. Local wear leveling is invoked after a threshold number of writes to the frame. By using an algorithm like Start-Gap [26], wear leveling at this level can keep writes uniformly distributed within a frame. The second level uses global wear leveling across frames. Global wear leveling is invoked after a threshold number of total writes to the memory or may be triggered when extreme skew in the writing patterns is detected. The goal of global wear leveling is to distribute writes evenly across frames. Wear leveling at this level constructs the optimal block migration plan based on the current wear distribution of the frames and the predicted demand distributions. By default, we use the past accesses to predict the access distributions of the next global wear-leveling period. Two optimizations, adaptive pruning and randomization, are applied to the cycle decomposition of the permutation defining the migration. These optimizations reduce the number of block movements and avoid destructive repetitive patterns that accelerate wearout. Our results show that Ouroboros can distribute writes uniformly across the whole NVRAM with low overhead and is robust to attack by write patterns that easily circumvent previous wear-leveling methods.

We make the following contributions in this article:

- Propose a robust hierarchical wear-leveling framework combining local and global wear leveling. We use a Start-Gap-like approach for local wear leveling and a novel accessprediction-based smoothing strategy for global wear leveling.

- Devise a block migration strategy that deterministically smooths wear based on access prediction, while using randomization to avoid destructive write patterns when the prediction is incorrect. The method is a hybrid between deterministic and randomized methods that achieves the best of both strategies. Specifically, it has the performance of an optimal deterministic migration strategy when the prediction is correct, while doing no worse than a randomized scheme when the prediction is incorrect. In contrast, purely deterministic schemes [35] perform poorly under bad conditions, while purely randomized schemes [9] fail to exploit optimization opportunities in their desire to avoid any bad cases.

- Formalize a method to set parameter values based on time and space overhead limitations and specified target smoothness levels.

- Perform evaluations on synthetically generated microbenchmarks, storage traces, and memory traces, and demonstrate the usefulness of the Ouroboros approach.

The remainder of the article is organized as follows. Section 2 introduces the motivation for the hierarchical wear-leveling method and proposes a novel design for block migration. Section 3 explains our Ouroboros wear-leveling approach and its optimizations. Section 4 describes possible implementations of the approach at the level of an NVRAM controller. Section 5 describes the experiments and their evaluations. In Section 6, overheads caused by wear leveling are also analyzed and the method to choose optimized parameter settings is discussed. Finally, a summary is presented in Section 7.

\section{MOTIVATION}

In this section, we analyze three basic policies for wear leveling: DUSS [35], RUSS [9], and Deterministic Demand-based Segment Swap (DDSS) on two specialized memory access traces. The analysis motivates the Ouroboros block migration policy, which forms the basis of our wear-leveling approach. 


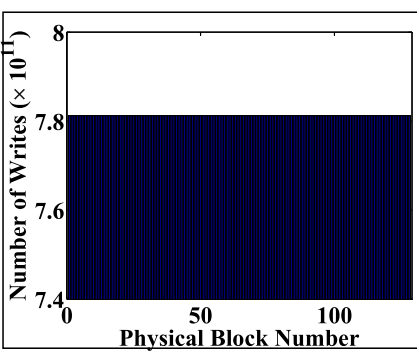

(a) DDSS (A* pattern)

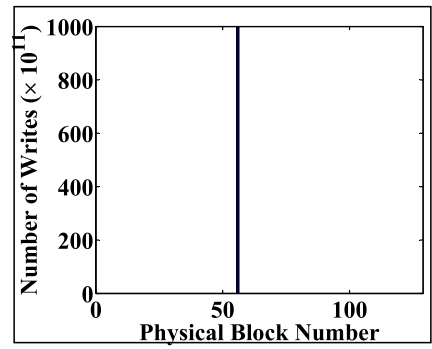

(d) DDSS (AB* pattern)

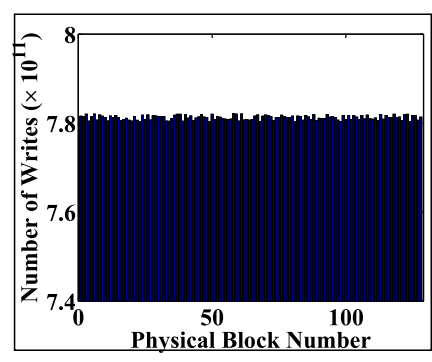

(b) DUSS (A* pattern)

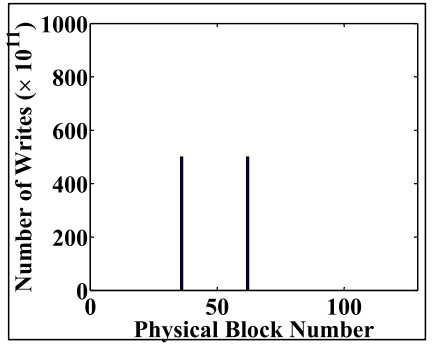

(e) DUSS (AB* pattern)

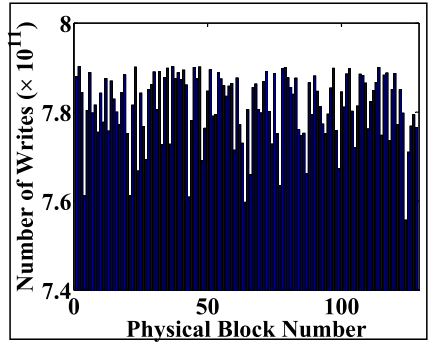

(c) RUSS (A* pattern)

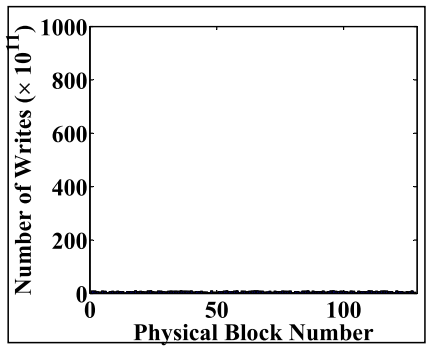

(f) RUSS (AB* pattern)

Fig. 1. Usage distribution for $A^{*}$ pattern and $A B^{*}$ pattern with DDSS, DUSS, and RUSS policies.

Usage-based policies [9, 15, 33, 35] maintain the usage count (accumulated number of writes) of every frame. Block reorganization is triggered after a threshold number of writes have been made to the memory space since the last reorganization. The period between reorganizations is referred to as an epoch. DUSS deterministically swaps the block in the highest-usage frame with the block in the lowest-usage frame. RUSS swaps the blocks in the highest-usage frame with a randomly selected frame. DDSS is similar to DUSS with one important difference: the block chosen for swapping is the one that is predicted to have the maximum access (number of writes) in the next epoch. This predicted high-access block is swapped with the block in the lowest-usage frame. We employ a simple predictive scheme that uses the memory access pattern in the previous epoch as the prediction of the memory access pattern in the next epoch. In the following section, DUSS, RUSS, and DDSS are analyzed for two synthetically generated memory traces: $A^{*}$ Write Pattern and $A B^{*}$ Write Pattern. The analysis clearly demonstrates the advantages and drawbacks of the three wear-leveling approaches.

\subsection{Analysis of Simple Reorganization Policies}

$A^{*}$ Write Pattern: Consider the write pattern that continuously writes to a single logical block A. We refer to this write sequence as the $A^{*}$ pattern. We start with an initial random distribution of writes on the frames and then apply a sequence of $10^{14}$ writes to a logical block $A$. The usage distribution for the $A^{*}$ pattern under the DDSS policy is shown in Figure $1(a)$. For the $A^{*}$ pattern, the predictor will always make a correct prediction of the access. Therefore, the usage distribution is very smooth, with all frames getting roughly the same usage. Figure 1(b) shows the usage distribution of the $A^{*}$ pattern using DUSS. The smoothness of its usage distribution is similar to that of the DDSS policy. However, the usage distribution of the $A^{*}$ pattern using the RUSS policy, which is shown in Figure 1(c), is not that smooth. 


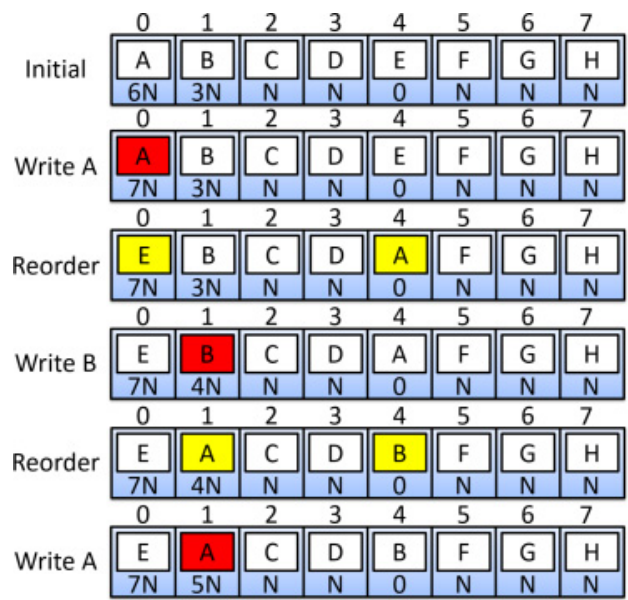

(a) Failure of DDSS under (AB)* Pattern.

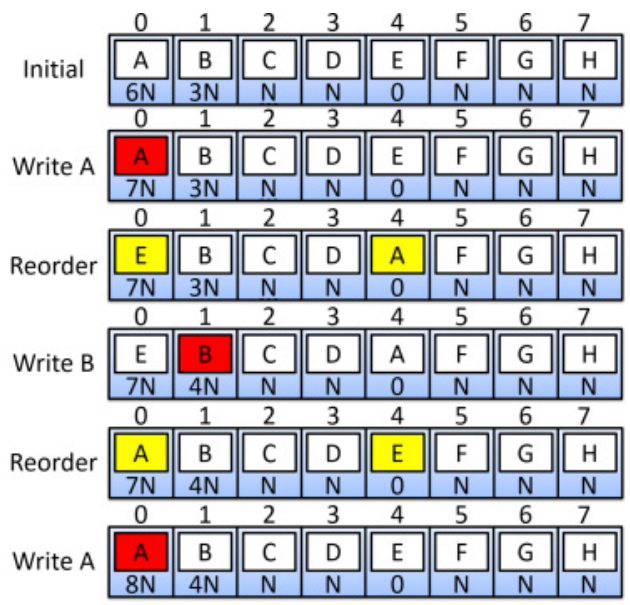

(b) Failure of DUSS under (AB)* Pattern.

Fig. 2. Memory behavior for DDSS and DUSS under (AB)* pattern.

$\mathrm{AB}^{*}$ Write Pattern: The $\mathrm{AB}^{*}$ pattern alternates writes between two blocks $\mathrm{A}$ and $\mathrm{B}$ in every epoch. In odd-numbered epochs, all writes are to block $A$, and in even-numbered epochs, all writes are to block B. In this case, access prediction (which uses the write distribution in the current epoch as the prediction for the next epoch) will fail every time. Hence, this represents a worst-case pattern for the deterministic demand-based policy DDSS. The behavior of DDSS is shown in Figure 2(a). Reorganization is assumed to happen after every $\mathrm{N}$ writes. The numbers under each frame indicate the current usage of that frame. After the first $\mathrm{N}$ writes to $\mathrm{A}$, the predictor will (wrongly) predict that $\mathrm{A}$ will be written again in the next epoch and swap block $\mathrm{A}$ in frame 0 with block $\mathrm{E}$ in the lowest-usage frame 4 . The next $\mathrm{N}$ writes are to block $\mathrm{B}$ in frame 1 , and the next swap will exchange the predicted high-demand block B in frame 1 with block A in the lowest-usage frame 4 . A and B continually ping-pong between frame 1 and frame 4 . The block that will actually be written in that epoch will always be in frame 1 . Hence, all writes will continue without bound on the highest-usage frame. The spike causing wearout can be clearly seen in Figure 1(d).

A similar destructive write pattern emerges for DUSS as well. The behavior of DUSS is shown in Figure 2(b). After $\mathrm{N}$ writes to block $\mathrm{A}$, a reorganization is triggered. The highest- and lowestusage frames ( 0 and 4 , respectively) swap their contents. The next $\mathrm{N}$ writes to $B$ increases the usage of frame 1 to $4 N$. However, the reorganization will again swap the contents of frames 1 and 2 , bringing block $A$ back to its initial location in frame 0 . This process will continue indefinitely: all writes to $A$ will be made to frame 0 and all writes to $B$ to frame 1 . This results in a distribution with two spikes corresponding to the writes to these two frames as is shown in Figure 1(e). The wear-leveling swap steps are ineffective in preventing the concentration of the writes. Finally, Figure 1(f) shows the usage distribution for the $(\mathrm{AB})^{*}$ pattern using the randomized RUSS policy. It shows that by involving randomization, the drawbacks of a deterministic swap are removed.

\subsection{New Block Migration Policy}

The problems in handling the $\mathrm{A}^{*}$ and $\mathrm{AB}^{*}$ patterns described previously motivates our new migration policy. As shown by the deterministic swap methods (DUSS and DDSS) for the $\mathrm{A}^{*}$ pattern, we can benefit tremendously over random swap (RUSS) by using knowledge of future accesses in determining where to migrate a block. On the other hand, the $\mathrm{AB}^{*}$ pattern shows that incorrect 
Table 1. Wear-Leveling Method Comparison

\begin{tabular}{|l|l|l|l|}
\hline Methods & Table Support & WL Basic Policy & Block Migration Rule \\
\hline \hline Start-gap [26] & No & Circular rotation & Random \\
\hline Curling-pcm [22] & No & Circular rotation & Deterministic \\
\hline Segment swap [35] & Yes & Usage based & Deterministic \\
\hline PCM-aware swap [9] & Yes & Usage based & Random \\
\hline Unit bin [3] & Yes & Demand based & Deterministic \\
\hline Ouroborous & Yes & Demand based & Hybrid \\
\hline Methods & Hardware Support & Online & Application \\
\hline \hline Start-gap [26] & Controller level & Online & General \\
\hline Curling-pcm [22] & Controller level & Online & Embedded system \\
\hline Segment swap [35] & Chip level & Online & General \\
\hline PCM-aware swap [9] & Chip level & Online & General \\
\hline Unit bin [3] & Controller level & Offline & General \\
\hline Ouroborous & Controller level & Online & General \\
\hline
\end{tabular}

prediction in a deterministic strategy can have severe performance consequences, which are avoided by a randomized approach. We propose a hybrid solution that exploits correct predictions when possible to obtain an optimal migration strategy while simultaneously guarding against incorrect predictions by using randomization to avoid worst-case behavior. Thus, we obtain the best-case performance of an optimal deterministic strategy and a worst-case performance of a randomized scheme. Our solution is to add a small degree of randomization: one random block is included in each deterministic block migration to obfuscate either an adversary or a deterministic worst-case pattern. The solution is described in detail in Section 3.3.

\subsection{Previous Work}

A large number of wear-leveling methods specifically for NVRAM [6, 7, 11, 14, 15, 22, 24, 26, 30, 32, 35] have been proposed over the last decade. A comparison of the most popular and representative wear-leveling methods specialized for NVRAM is shown in Table 1.

First, the wear-leveling methods are distinguished by their use of either table-based or algebraicbased mappings as discussed in Section 1. Start-Gap [26] and Curling-PCM [22] are examples of algebraic-based mappings. The methods are also distinguished by how they choose which data to migrate. Algebraic methods use simple static rules that migrate memory lines or blocks in a predetermined order (usually circular shifting). Other methods decide to migrate a block based on the usage of the frame it occupies $[9,35]$, while [3] and Ouroboros use the number of future writes to a logical block (called its demand) in choosing migration candidates. A third axis for differentiation is based on how the target frame for a migration is chosen: deterministic migration, random migration, or hybrid migration. Deterministic migration [3, 22, 35] guarantees a smooth wearout when the assumptions on which the wear-leveling method is based are met, but can have catastrophic consequences when faced with different circumstances. Furthermore, deterministic wear-leveling methods face the possibility of security attacks whereby attack access patterns can be devised to negate wear leveling based on deterministic migration rules. Randomized migration [9] addresses this situation by choosing all destination frames randomly. This obfuscates wear-leveling attacks, but also discards useful access information provided implicitly or explicitly by an application, essentially reducing the best and the worst cases to the same level. Start-Gap [26] uses a cryptographically secure three-stage Feistel Network mapping to randomize the locations of memory 
lines. Only Ourobouros explicitly combines the advantages of both deterministic and randomized migration to design a hybrid migration policy.

Most of the wear-leveling methods only require system support for wear leveling at the controller level. However, Segment swap [35] and PCM-aware swap [9] also perform wear leveling at fine granularity, which requires chip-level hardware support. Finally, Curling-pcm [22] is designed specifically for embedded system environments where application memory accesses can be completely profiled and the data layout in memory can be controlled to match the needs of the wear-leveling scheme. This works well in that environment, but cannot be used in a general memory or storage environment, where data layout and access patterns are not controlled. Finally, Unit bin [3] is an offline algorithm for constructing an optimal migration schedule. The algorithm requires precise prediction of all future memory accesses, which is not available in practice.

\section{OUROBOROS WEAR LEVELING}

In this section, we describe the details of two-level hierarchical Ouroboros wear leveling and its optimization.

\subsection{Overview}

The NVRAM is modeled as an array of memory cells accessed by the processor using logical addresses. The logical address is intercepted by the NVRAM controller and mapped to an internal physical address within the memory chips from where the data is accessed. By changing the logicalto-physical mapping, writes to the same logical address can be redirected to different memory cells at different times, thereby reducing excessive writes of any physical location. In this article, we refer to memory writes to the physical address space as usage and memory writes to the logical address space as demand. Wear leveling attempts to equalize the usage of the memory cells by changing the mapping between logical and physical locations dynamically.

The logical address space is made up of $N$ blocks. Blocks are made up of memory lines. The NVRAM is likewise partitioned into physical frames. A frame holds a block and an additional memory line (called the "GapLine") for local wear leveling. A logical block can be placed in any physical frame and the fully associative mapping is maintained in a frame table saved persistently in the NVRAM.

We propose a two-level mapping scheme (local and global wear leveling) to relocate cells for wear leveling. Local wear leveling works within each frame to keep the usage of memory cells within it balanced. Global wear leveling works across frames to balance their usage. The two schemes operate more or less independently at different spatial and temporal granularities. The logical address is split into a logical block number (LBN) and a logical offset. The frame table translates the LBN to a physical frame number (PFN). The logical offset within the block is translated to a physical offset within the frame by an easy-to-compute mapping that accounts for the local wear leveling [26] within the frame.

Global wear leveling occurs at epoch boundaries. When the total number of writes to the NVRAM since the last epoch exceeds a global threshold $\left(\Gamma_{G}\right)$, it triggers a reorganization event: a subset of blocks are chosen and migrated to different frames in an attempt to even the wear of the frames. During an epoch, local wear leveling continues within each frame. Specifically, when the number of writes to a frame exceeds a local threshold $\left(\Gamma_{L}\right)$, a single memory line within the frame is relocated to a free line in the frame (the "GapLine" in Start-Gap wear leveling [26]).

\subsection{Local Wear Leveling}

For local wear-leveling, we implement a Start-Gap-like [26] technique. For each frame, we have an additional empty line serving as the GapLine. When the number of writes to the block reaches the 
local threshold, the content of the line adjacent to the GapLine is copied to it. However, instead of randomizing the memory lines across the entire memory as in [26], we use a simple static random permutation of the address within the blocks. ${ }^{1}$ This module is not described here and the reader is referred to the original paper [26] for details. Using Start-Gap, after every $\Gamma_{L}$ (local threshold) writes to a frame, one memory line is relocated to an adjacent memory line in the frame in a circular, round-robin manner. Hence, if there are $N_{L}$ lines in a block, then every memory line is guaranteed to be relocated after $N_{L} \times \Gamma_{L}$ writes to the block. In the worst case, a single memory line may also get $N_{L} \times \Gamma_{L}$ writes before it is relocated. Local wear leveling evenly distributes writes within a frame and reduces the frequency of global wear leveling.

\subsection{Global Wear Leveling}

For global wear leveling we use Algorithm 1 to distribute writes smoothly across frames. In the following sections, the basic algorithm without optimization is presented, followed by a description of the pruning (Algorithm 2) and randomization (Algorithm 3) optimizations.

ALGORITHM 1: Global Wear Leveling: Basic Algorithm

Step 1

- Sort blocks in decreasing order of demand vector $P$.

- Sort frames in increasing order of usage vector $U$.

- Let the block residing in frame w be $\beta(w)$.

Step 2 Generate raw migration mapping $\pi$ :

- Map block with $i^{\text {th }}$ highest demand to the frame with $i^{\text {th }}$ lowest usage.

- Call the $K$ blocks with highest demand as hot and the remaining blocks as cold.

- Denote the set of $K$ hot blocks as $\mathcal{H}$ and the set of $N-K$ cold blocks as $C$.

Step 4 Pruning: Construct a set of pruned sequences $\Sigma^{\prime}$ that describe the migration schedules of the hot blocks induced by themapping $\pi$. Details are shown in Algorithm 2.

Step 5 Randomization: For each sequence $\Sigma^{\prime}$ generated in Step 4, create an augmented sequence $\Sigma$ by adding one randomly chosen block to $\Sigma^{\prime}$. Details are shown in Algorithm 3.

Step 6 Perform the block migrations:

- For each augmented sequence $\Sigma$ from Step 5:

- If $\Sigma=\left(h_{1}, \pi\left(h_{1}\right), h_{2}, \pi\left(h_{2}\right), \ldots, h_{i}, \pi\left(h_{i}\right), \ldots, h_{k}, \pi\left(h_{k}\right), c, r, \beta(r), \beta^{-1}\left(h_{1}\right)\right)$, perform the migrations specified: Move block $h_{i}$ to frame $\pi\left(h_{i}\right), 1 \leq i \leq k$, move block $c$ to frame $r$, and move the block in frame $r$ to the frame initially occupied by $h_{1}$.

\section{Basic Algorithm}

The demand of a block is a prediction of the number of writes that will be made to it in the next epoch. The demands of the blocks are represented by the vector $\mathbf{P}=\left[p_{1}, p_{2}, \cdots p_{N}\right]$. We define the usage $u_{i}$ of a physical frame $i$ to be the cumulative number of writes that have been made to the frame. The goal of the global wear leveling is to make the smoothest usage vector $\mathbf{U}=$ $\left[u_{1}, u_{2}, \ldots u_{N}\right]$.

\footnotetext{
${ }^{1}$ This is needed to thwart security attacks that exploit the deterministic nature of local wear leveling to create adversarial write patterns.
} 


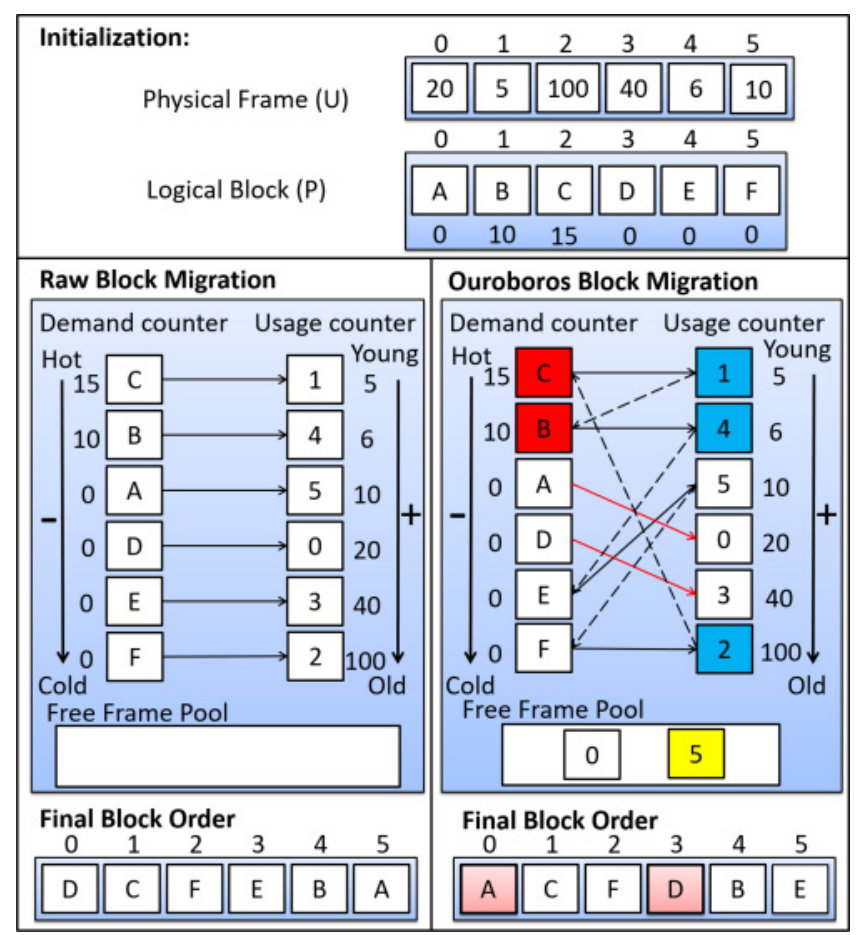

Fig. 3. Demand-based global wear leveling.

For the reorganization we define the mapping $\pi$ that maps the block with the $i^{\text {th }}$ highest demand to the frame with the $i^{t h}$ lowest usage. This mapping results in the lexicographically minimum ${ }^{2}$ usage vector at the end of the next epoch, that is, the smoothest usage distribution possible starting from the current usages and projected demands.

Example: Raw Block Migration (see Figure 3 left panel). Suppose we have six blocks A, B, $\mathrm{C}, \mathrm{D}, \mathrm{E}$, and $\mathrm{F}$ that are initially mapped to physical frames 0 through 5 , respectively. The projected demand vector is given by $\mathbf{P}=[0,10,15,0,0,0]$ and the usage vector is given by $\mathbf{U}=$ $[20,5,100,40,6,10]$. Arranging the blocks in decreasing demand order, we get the sequence: $\mathrm{C}$, $\mathrm{B}, \mathrm{A}, \mathrm{D}, \mathrm{E}, \mathrm{F}$. Arranging the frames in increasing usage order, we get the sequence: $1,4,5,0,3$, 2. The mapping $\pi$ is as follows: $\pi_{1}(C)=1, \pi_{2}(B)=4, \pi_{3}(A)=5, \pi_{4}(D)=0, \pi_{5}(E)=3, \pi_{6}(F)=2$. Hence, $C$ is moved to frame 1, displacing its current occupant, which is block B. Since $\pi_{2}(B)=4$, $\mathrm{B}$ is moved to frame 4, displacing its occupant $\mathrm{E}$, which is now moved to frame $\Pi_{5}(E)=3$. The current occupant of frame 3 is $\mathrm{D}$, which is relocated to $\pi_{4}(D)=0$, displacing $\mathrm{A}$, which is moved to $\pi_{3}(A)=5$, displacing its occupant $\mathrm{F}$, which is moved to $\pi_{6}(F)=2$. The cycle is complete, resulting in a cyclic shift of the blocks (C, B, E, D, A, F) or equivalently the frames $(2,1,4,3,0,5)$. The final usage of frames based on the projected demand will be as follows: frame 0: $20+0=20$, frame 1 : $5+15=20$, frame $2: 100+0=100$, frame $3: 40+0=40$, frame $4: 6+10=16$, frame $5: 10+0=10$. Hence, the predicted usage vector $\boldsymbol{U}$ at the end of the next epoch is $[20,20,100,40,16,10]$, which is the smoothest vector possible based on the projected demand and current usage distribution.

\footnotetext{
${ }^{2}$ The lexicographically smoothest vector will have the smallest maximum element, and among the vectors with the same maximum it has the second smallest maximum, and so on.
} 


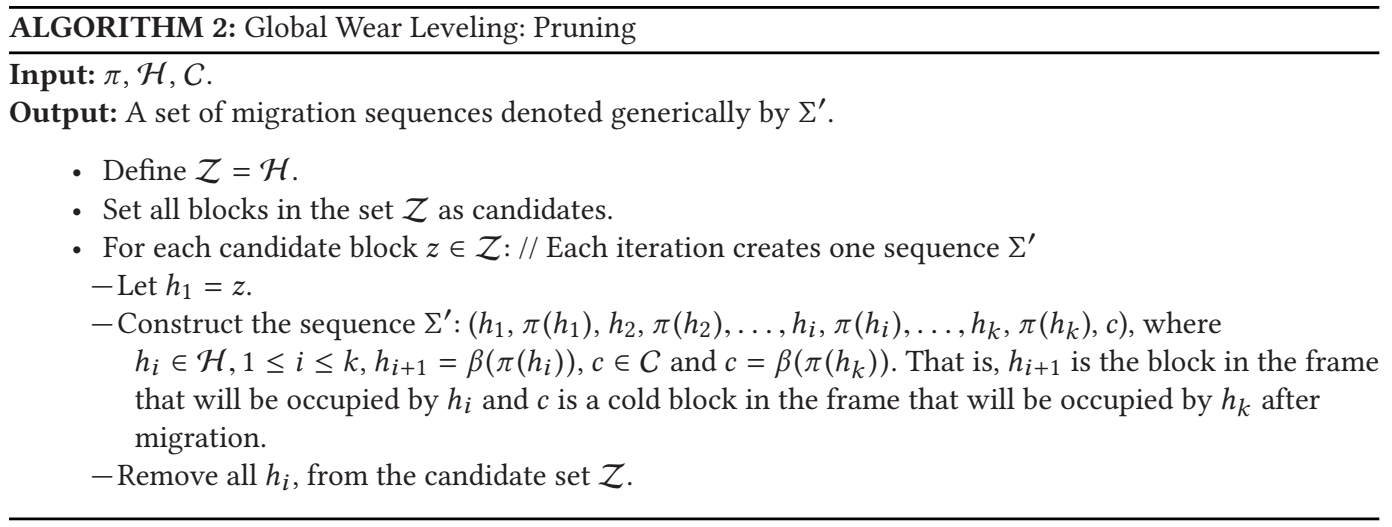

ALGORITHM 3: Global Wear Leveling: Randomization

Input: Sequence $\Sigma^{\prime}=\left(h_{1}, \pi\left(h_{1}\right), h_{2}, \pi\left(h_{2}\right), \ldots, h_{i}, \pi\left(h_{i}\right), \ldots, h_{k}, \pi\left(h_{k}\right), c\right)$, obtained from Algorithm 2. Output: Cyclic Sequence $\Sigma=\left(h_{1}, \pi\left(h_{1}\right), h_{2}, \pi\left(h_{2}\right), \ldots, h_{i}, \pi\left(h_{i}\right), \ldots, h_{k}, \pi\left(h_{k}\right), c, r, \beta(r), \beta^{-1}\left(h_{1}\right)\right)$, obtained by augmenting $\Sigma$ with a free frame and completing the cycle.

Define: $\eta$ to be the set of all the frames of the memory.

Define: $\mu(H)=\left\{\pi\left(h_{i}\right) \mid \forall h_{i} \in \mathcal{H}\right\}$ to be the set of new frames of the hot blocks after migration.

Define: $v(H)=\{\mathrm{f} \mid \beta(f) \in \mathcal{H}\}$ to be the set of frames currently occupied by hot blocks.

- Construct the free frame set $\mathcal{R}=\eta-\{\mu \cup v\}$.

- Map the cold block $c$ to a randomly chosen frame $r \in \mathcal{R}$.

- Map the block currently in frame $r(\beta(r))$ to the frame currently occupied by $h_{1}\left(\beta^{-1}\left(h_{1}\right)\right)$, thus completing the migration schedule $\Sigma=\left(h_{1}, \pi\left(h_{1}\right), h_{2}, \pi\left(h_{2}\right), \ldots, h_{i}, \pi\left(h_{i}\right), \ldots, h_{k}, \pi\left(h_{k}\right), c, r, \beta(r)\right.$, $\left.\beta^{-1}\left(h_{1}\right)\right)$.

\section{Optimization Details}

Consider the behavior of the raw block migration mapping in the basic algorithm. Even though the raw block migration mapping guarantees the smoothest usage vector, the direct use of the mapping would imply a reshuffling of all the blocks in the NVRAM, a costly and impractical requirement. Moreover, the blocks with small demand values will not affect the usage distribution significantly. Hence, at each reorganization period, we settle for shuffling at most $K$ highest-demand blocks and save the overhead of migrating blocks with low demand. This is the pruning operation in Algorithm 2. The second issue is that this mapping is completely deterministic (based only on the sequence of past accesses) and vulnerable to attack with bad patterns (like the $\mathrm{AB}^{*}$ sequence discussed in Section 2.1). We address this issue by introducing a small degree of randomization in each cyclic shift of blocks to emulate the Ouroboros block migration ring in Algorithm 3.

Pruning Operation: In order to adaptively prune the number of blocks to be migrated at the end of an epoch, we select a group of blocks with high predicted demands into a $\operatorname{Hot} \operatorname{Block} \operatorname{Pool}(\mathcal{H})$. The predicted demand for block $i$ is based on the demand to the block since its last migration and is maintained in a counter $P_{i}$. A block $i$ is hot if $P_{i}$ exceeds a specified threshold. The Hot Block Pool is made up of up to $K$ hot blocks with the highest predicted demands. The choice of the parameter $K$ will be discussed later. The blocks in the Hot Block Pool are marked as candidates for migration and the mapping $\pi$ specifies their destination frames. The blocks currently resident in these destination frames will also need to be migrated and are marked as well. Since these two sets of marked blocks may overlap, the total number of marked blocks will be between $K$ and $2 K$. 


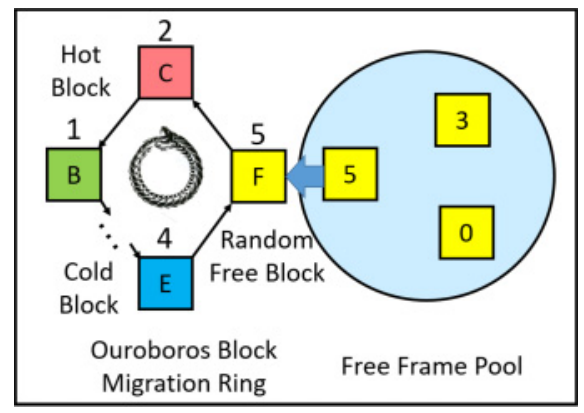

Fig. 4. Ouroboros block migration rule.

Limiting the number of block migrations in a period helps us lower the time overhead required for block reorganization. However, there can be situations (albeit rare) when a large number of blocks simultaneously become hot. This can happen if the demand for these blocks all grow uniformly for a number of epochs and they all cross the threshold simultaneously. Therefore, by only moving $\mathrm{K}$ hot blocks, the remaining hot blocks may continue to accumulate writes before getting their turn to migrate. In order to prevent this special case, we record the number of global wearleveling periods for which a hot block has not been migrated. These blocks will be given priority during the next migration.

The number of blocks that need to be moved to new locations during a migration lies somewhere between $K$ and $3 K$ depending on the location of the hot and cold blocks in their physical frames. The time allocated for performing the migration places an upper bound on $K$. A secondary constraint that can theoretically limit the number of block migrations $K$ is the number of free frames in the Free Frame Pool $(\mathcal{R})$. The Free Frame Pool is used to introduce randomization in global wear leveling. The construction of the Free Frame Pool is described in detail in the Randomization Operation section later. There should be enough free frames to complete every cycle. In the worst case, there are $K$ cycles (each cycle involving one hot and one cold block) requiring $K$ free frames. Also, the number of frames used by marked blocks in this case will be $2 K$. Hence, we require $K$ to be no more than one-third of the total number of frames. In practice, this will not be the limiting reason since in an epoch we migrate only a small percentage of the total frames.

Example: Ouroboros Block Migration 1 (see Figure 3 right panel). The permutation $\pi$ between blocks and their new frame locations is the same as that in the Raw Block Migration example described earlier. However, now we assume that blocks $\mathrm{C}$ and $\mathrm{B}$ are in the Hot Block Pool, and the Free Frame Pool is $\{0,5\}$. Starting from hot block C, we have $\pi_{1}(C)=1$. Since the block currently in frame 1 is a hot block $\mathrm{B}$, we continue the cycle. Now $\pi_{2}(B)=4$ and the block currently in frame 4 is a cold block E. Thus, the first sequence $\Sigma^{\prime}$ constructed after Step 4 is $(C, 1, B, 4, E)$. Since there are no other hot blocks, we have only one sequence $\Sigma^{\prime}$.

Randomization Operation: To select a random free frame in the Ouroboros block migration ring, we maintain a pool of frames called the Free Frame Pool $(\mathcal{R})$. Frames in this pool must not hold any of the blocks currently marked for migration. The Free Frame Pool chooses more than K frames with the lowest usage from the frames holding unmarked blocks.

To create the migration schedule, we proceed as shown in Figure 4. Starting from a hot block $(C)$, we follow the cycle induced by the permutation $\pi$ till we encounter a cold block $(E)$ that needs to be moved to make space for an incoming hot block. Now, rather than closing the cycle by moving the cold block to the frame occupied by the initial hot block $(C)$, we instead close the cycle after adding one randomly chosen frame (F) from the Free Frame Pool. 


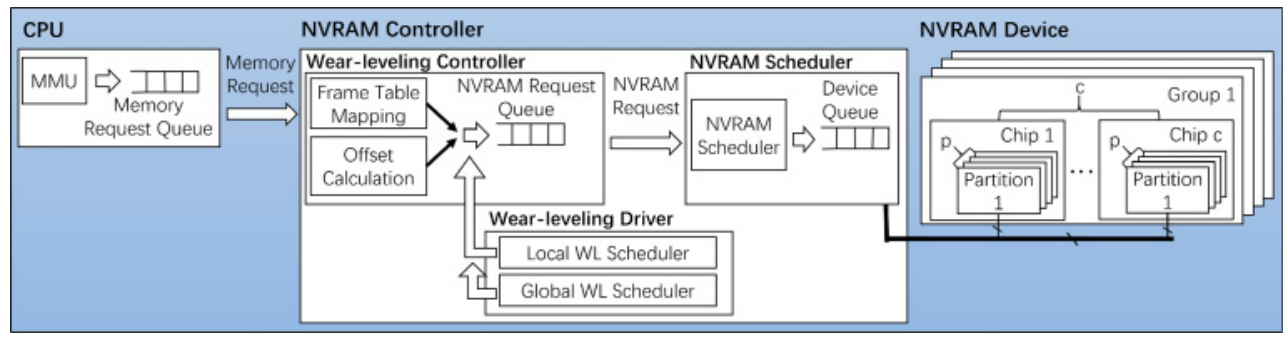

Fig. 5. Architecture of NVRAM memory system.

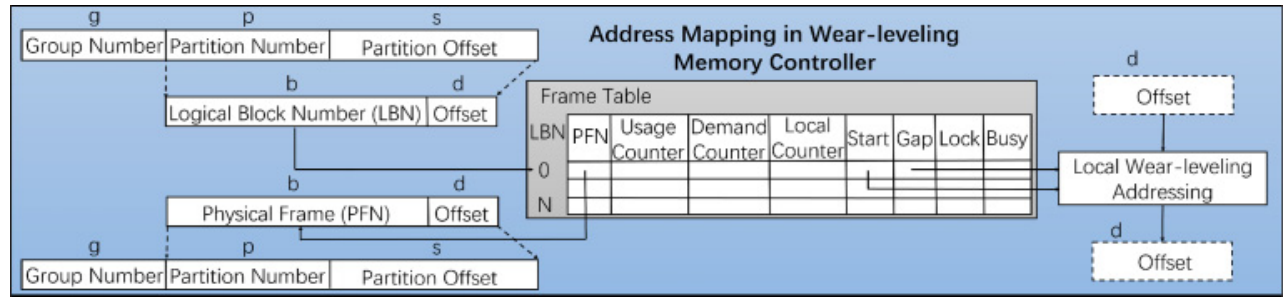

Fig. 6. Frame table mapping.

Example: Ouroboros Block Migration 2. We continue the example by preparing to close the sequence $\Sigma^{\prime}$ in step 5 (Algorithm 3 ) by augmenting the sequence $\Sigma^{\prime}$ with a frame randomly chosen from the Free Pool Frame. In the example, suppose we choose frame 5 . We move $\mathrm{E}$ to frame 5 and the current block in frame 5 back to the location of the initial hot block $\mathrm{C}$, which is frame 2 . The augmented sequence is now $(C, 1, B, 4, E, 5, F, 2)$. The Ouroboros block migration ring is complete, resulting in a cyclic shift of the blocks $(\mathrm{C}, \mathrm{B}, \mathrm{E}, \mathrm{F})$ or equivalently the frames $(2,1,4,5)$. The final usage of frames based on the projected demand in this case will be the same as that found for the raw block migration. The usage vector $\mathbf{U}$ for the next epoch is also $[20,20,100,40,16,10]$, which is the lexicographically smallest vector possible based on the projected demand and current usage.

\section{DESIGN OF NVRAM MEMORY SYSTEM}

In this section, we discuss the implementation of our Ouroboros wear-leveling framework in a system with NVRAM devices. The architecture of the NVRAM device is described along with the high-level implementation of the NVRAM controller.

\subsection{NVRAM Device Model}

An NVRAM device has an internal architecture similar to a DRAM DIMM (Figure 5). A typical organization consists of $2^{g}$ groups; each group is made up of $2^{c}$ chips and each chip has $2^{p}$ partitions. There are $2^{s}$ memory lines each of size $2^{r}$ bytes in every partition. The total size of the NVRAM device is $2^{m}$ bytes $(m=g+p+s+c+r)$. NVRAM requests (reads/writes) have a granularity of $2^{r+c}$ bytes and are fully striped at the same memory line address across all the chips in a group; hence, the address for the NVRAM device is $g+p+s$ bits. Due to this striping, the whole memory can be managed by considering just one chip. As shown in Figure 6, the $2^{p+s}$ address range of a group in one chip is broken up into a LBN and a block offset for global wear leveling.

We perform global wear leveling independently in each group. This is because the NVRAM typically services requests in different groups concurrently, and applications with predictable data access patterns exploit this parallelism when making data layout decisions. Dynamically moving 
blocks across groups may destroy this natural concurrency. However, we note that it is possible to extend the domain of wear leveling across groups by including placement and data affinity (antiaffinity) information in computing a cross-group migration schedule. This extension is not addressed in this article.

\subsection{Implementation}

In this section, the details of the NVRAM storage system addressing and its operation with the local and global wear-leveling mechanisms are described.

Emerging programming models exploit the byte addressability of NVRAM storage by memory mapping its regions directly into the application's virtual address space [13]. This is different from traditional SSD block accesses, which are made through the IO subsystem. We assume a memorymapped implementation.

The CPU address output after MMU translation (Figure 5) is the logical address for the NVRAM. A wear-leveling module in the NVRAM controller performs the translation to physical NVRAM addresses, similar to the Flash translation layer (FTL) in an SSD controller. It translates the logical block number to the physical frame number within the NVRAM device and the logical offset of the block to the physical offset of the frame. The frame table (Figure 6) must be stored persistently in the NVRAM and updated as block migrations are made. In order to speed up translation, the currently used portions of the frame table are cached in SRAM available to the controller. The frame table stores the mapping of a LBN to the PFN. The wear-leveling module divides the address within a group into the LBN and logical block offset. By looking up the frame table, the LBN is replaced with PFN and the logical block offset is mapped to the physical frame offset through local wear-leveling addressing. The PFN and physical block offset are concatenated along with the group and chip fields to obtain the physical address (PA).

The frame table holds descriptors for each logical block; each descriptor maintains several flags and counters to represent the state of its block. The DEMAND COUNTER accumulates the number of writes made to a block before migration. It is used to predict the demand for this logical block in the next epoch of global wear leveling; the counter is incremented when the block is written to and cleared when the block is migrated.

The LOCAL COUNTER is used to trigger a local wear leveling of the block. It can be implemented as the lower-order bits of the USAGE COUNTER. The START and GAP pointers that are related to the local wear leveling are also stored with the LOCAL COUNTER to track the start and gap position in each physical frame. The USAGE COUNT tracks the number of writes to the physical frame in the PFN field. When a block is migrated, the descriptor of the new logical block that is moved to the physical frame is updated with its USAGE COUNT. This organization allows updating the frame usage during the lookup of the frame table. The counters in the frame table provide the input vectors to the wear-leveling algorithm.

We use two synchronization bits (LOCK and BUSY) for each block to prevent inconsistency during block migration. The BUSY flag is set to indicate that an access to the block is in progress. The LOCK flag is used to indicate that a migration of the block out of or into that frame is in progress. When the wear-leveling driver needs to copy the contents into or out of a frame, it sets the LOCK flag in the frame table entry to 1 and waits for the BUSY flag to be 0 . A 0 BUSY flag indicates that no one is accessing that frame, and the wear-leveling read/write requests generated by the wear-leveling driver can safely copy content into or out of that frame. If the BUSY flag is 1 , wear leveling needs to wait for it to be cleared to 0 . Before an access is allowed to a block, the LOCK bit is examined; if set, the access is delayed until LOCK is reset to zero.

After the physical address calculation, the original memory request is rewrapped into a standard NVRAM request for the scheduler. The NVRAM scheduler is also part of the NVRAM controller. 
The NVRAM scheduler gets NVRAM requests and dispatches them to the NVRAM device according to its internal policy consistent with the command timing constraints of the device (e.g., read-to-read delay, read-to-write delay, write-to-write delay, and write-to-read delay).

For the implementation of the wear leveling, the wear-leveling driver in the NVRAM controller triggers both local wear leveling and global wear leveling. The local wear-leveling scheduler is woken up when the LOCAL COUNTER of any frame reaches the local wear-leveling threshold $\left(\Gamma_{L}\right)$. After being woken up, the local wear-leveling scheduler generates local wear-leveling read and write requests to copy a memory line into the GapLine. Those requests are put into the NVRAM request queue and START and GAP pointers are updated in the frame table.

Similar to the local wear-leveling scheduler, the global wear-leveling scheduler is woken up when the total number of writes to the NVRAM since the last global wear leveling exceeds the defined global threshold $\left(\Gamma_{G}\right)$. After being woken up, the scheduler will generate the permutation $\pi$ and the block reorganization schedule as described in Section 3.3. Then the blocks are migrated one at a time following the schedule. For each block migration, the scheduler will generate a group of read and write requests and place them in the NVRAM request queue. The frame table will also be updated accordingly.

\section{EXPERIMENTAL EVALUATIONS}

We conducted several trace-driven experiments to evaluate the effectiveness of the Ouroboros wear-leveling method on three sets of benchmarks. The first set contains three microbenchmarks that can be compared against the analysis of the expected behavior. The aim of the microbenchmark experiments is to understand the behavior of each wear-leveling algorithm for certain write patterns with special characteristics on a small-sized NVRAM device. The second set is composed of storage traces from FIU and Microsoft. The third set is composed of two memory traces from CPU SPEC 2006. The aim of the those two real-life benchmark experiments is to evaluate the performance of each wear-leveling algorithm for realistic large NVRAM devices for both storage and memory. We also compare Ouroboros wear leveling with the DUSS and the RUSS.

\subsection{Smoothness Metrics}

Due to the limitations of having a single measure to distinguish the smoothness of different usage distributions, we present our results using several metrics. First, the actual usage distribution of the frames can give a visual view of smoothness. However, it cannot quantitatively allow comparison of different distributions. Therefore, we use a pair of measures, $l_{2}$ smoothness (standard deviation) and $l_{\infty}$ smoothness, as quantitative metrics that measure the deviation between the empirical usage vector and the theoretical ideal usage vector.

Suppose a total of $W$ writes are done on the NVRAM with $N$ frames. The actual usage distribution of the frames can be represented by the vector $\mathrm{U}=\left[u_{1} \cdots u_{N}\right]$ and the ideal (smoothest possible) write distribution can be represented by the vector in which all components $\widehat{u}_{i}$ are equal to the mean $W / N$. We use the $l_{2}$ smoothness (Equation (1)) and $l_{\infty}$ smoothness (Equation (2)) to measure the smoothness of the usage distribution:

$$
\begin{aligned}
& l_{2}=\sqrt{\frac{\sum_{i=1}^{N}\left(\frac{u_{i}-\widehat{u}_{i}}{W}\right)^{2}}{N}} \\
& l_{\infty}=\max _{i}\left|u_{i}-\widehat{u}_{i}\right| .
\end{aligned}
$$

The $l_{\infty}$ metric measures the maximum deviation of a component from the mean, while the $l_{2}$ metric is the standard deviation of the relative frequency distribution of the writes, that is, the standard deviation of the sequence $u_{i} / W, i=1 \cdots N$. The disadvantage of this metric is that 
Table 2. Parameters Settings

\begin{tabular}{|l|l|l|}
\hline Parameters & \multicolumn{1}{|c|}{ Micro } & Storage and Memory \\
\hline \hline NVM size $(\mathrm{M})$ & $512 \mathrm{MB}$ & $512 \mathrm{~GB}$ \\
\hline Frame size $(\mathrm{F})$ & $8 \mathrm{~KB}$ & $8 \mathrm{~KB}$ \\
\hline Line size $(\mathrm{L})$ & 16 bytes & 16 bytes \\
\hline Stripe size $(\mathrm{C})$ & 32 chips & 32 chips \\
\hline Local threshold $\left(\Gamma_{L}\right)$ & 195 & 195 \\
\hline Global threshold $\left(\Gamma_{G}\right)$ & $1 \times 10^{7}$ & $1 \times 10^{8}$ \\
\hline
\end{tabular}

the calculation tends to doubly penalize deviations from smoothness, since both deviations below the mean and deviations above the mean are used. For wear leveling, deviations below the mean are harmless as long as the mean itself is not changed significantly, but deviations above the mean can be dangerous. A larger $l_{2}$ or $l_{\infty}$ means a more uneven write distribution, while the smaller these values are, the closer is the usage distribution to the ideal.

\subsection{Experimental Setup}

For the experiment, we use the same NVRAM device model as described in Section 4.1 for simulation. In the NVRAM architecture described in Section 4.1, every memory request is striped across the chips in one group. For the microbenchmark experiments, we are interested in observing the behavior of the wear-leveling method on each frame. Therefore, we choose a small NVRAM of $512 \mathrm{MB}$ partitioned among 32 chips for a size of $16 \mathrm{MB}$ per chip. However, for the memory and storage experiments, the performance is evaluated with a more realistic memory size of $512 \mathrm{~GB}$ or 16 GB per chip.

The other memory parameters for the experiments are shown in Table 2. The frame size $\mathrm{F}$ is chosen to be $8 \mathrm{~KB}$ and the memory line size is 16 bytes to match the request size of 512 bytes striped across 32 chips. For the microbenchmark experiments, the trace consists of a total of $1 \times 10^{14}$ writes to the 512 MB NVRAM device. For the storage and memory benchmark experiments, we repeatedly feed the storage and memory traces to our NVRAM model until they complete 2,000 Ouroboros wear-leveling epochs. For the experiments with no wear-leveling, DUSS, and RUSS policies, we use the same number of writes as the Ouroboros experiments. The maximum size $K$ of the Hot Block Pool is set to be 10 . This means that no more than 30 blocks will be relocated during any global wear-leveling period. The global reorganization threshold $\Gamma_{G}$ and local reorganization threshold $\Gamma_{L}$ for the micro-, storage, and memory benchmarks are shown in Table 2. The rationale for these parameter values along with a design procedure to optimize their choice is described in Section 6.

\subsection{Usage Simulation Results}

In this section, we empirically compare the smoothness achieved by four different wear-leveling strategies for the microbenchmarks, storage traces, and memory traces. We compare smoothness both across frames (effectiveness of global wear leveling) and within frames (effectiveness of local wear leveling). For the interframe usage distribution, we show the distribution at the granularity of frames; each bar represents the total number of writes done to that frame. For the intraframe usage distribution, we show the distribution at the granularity of memory lines for a representative heavily used frame. The methods we compare in the experiments are No Wear Leveling, DUSS, RUSS, and Ouroboros. We use the two quantitative metrics $l_{2}$ and $l_{\infty}$ to compare these strategies. We also show the usage distribution of the frames using Ouroboros as a visual representation of the smoothness for all of the benchmarks. 


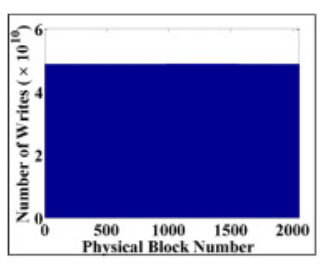

(a) $\mathrm{A}^{*}$ pattern.

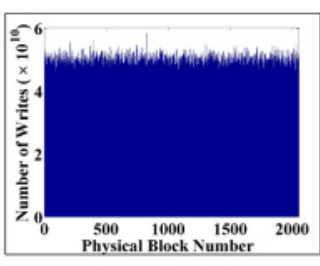

(b) $\mathrm{AB}^{*}$ pattern.

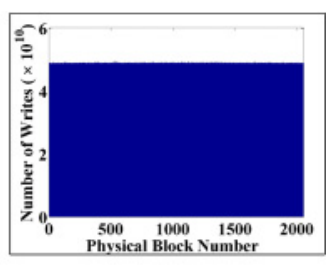

(c) $(\mathrm{AB})^{*} 50 \%$ pattern.

Fig. 7. Usage distribution across frames for microbenchmark traces after global wear leveling.

Table 3. Microbenchmark Measurements

\begin{tabular}{|l|l|l|l|l|l|l|}
\hline & \multicolumn{2}{|c|}{$\mathbf{A}^{*}$} & \multicolumn{2}{c|}{$(\mathbf{A B})^{*}$} & \multicolumn{2}{c|}{$(\mathbf{A B})^{*} \mathbf{5 0 \%}$} \\
\hline $\begin{array}{l}\text { Wear-Leveling } \\
\text { Method }\end{array}$ & $\begin{array}{c}\boldsymbol{l}_{2} \\
\left(\times 10^{-8}\right)\end{array}$ & $\begin{array}{c}\boldsymbol{l}_{\infty} \\
\left(\times 10^{6}\right)\end{array}$ & $\begin{array}{c}\boldsymbol{l}_{2} \\
\left(\times 10^{-5}\right)\end{array}$ & $\begin{array}{c}\boldsymbol{l}_{\infty} \\
\left(\times 10^{10}\right)\end{array}$ & $\begin{array}{c}\boldsymbol{l}_{2} \\
\left(\times 10^{-8}\right)\end{array}$ & $\begin{array}{c}\boldsymbol{l}_{\infty} \\
\left(\times 10^{7}\right)\end{array}$ \\
\hline \hline None & $2,200,000$ & $10^{8}$ & 1,600 & 5,000 & $1,600,000$ & $5,000,000$ \\
\hline DUSS & 1,290 & 2,260 & 1,600 & 4,950 & 2,960 & 517 \\
\hline RUSS & 6,980 & 29,200 & 5.77 & 2.89 & 5,710 & 2,040 \\
\hline Ouroboros & 3.9 & 8.13 & 1.3 & 4.88 & 8.17 & 7.19 \\
\hline
\end{tabular}

\section{Microbenchmarks}

The $\mathbf{A}^{*}$ pattern described in Section 2.1 is an extreme pattern of memory writes in which all the writes are concentrated to one block of the memory. This pattern has only write accesses and is the best case for the demand-based approach since its prediction of future accesses is always correct. Simulation results for the interframe usage distribution obtained by Ouroboros starting from a clear memory are shown in Figure 7(a). The smoothness of the distribution is visually clear in the figure. Furthermore, the $l_{\infty}$ and $l_{2}$ measures for Ouroboros, which are shown in Table 3, are significantly better than the metrics for both the deterministic (DUSS) and randomized (RUSS) usagebased methods. For this pattern, Ouroboros block migration greatly benefits from the consistently correct demand prediction; the usage distribution after wear leveling is extremely smooth, since it always does its writes to the lowest-usage frame. In this situation, the demand-based method migrates a block as soon as it gets too many writes. However, usage-based methods choose the blocks to migrate from among several aged blocks of similar usage and an incorrect choice does not help in smoothing. The randomized approach does somewhat worse than the deterministic approach, since the latter always moves a block to a low-usage frame, while the random selection used by RUSS can sometimes move a block to a more heavily used frame.

The $\mathbf{A B}^{*}$ pattern alternates its writes between two blocks in two successive epochs. This is also a pattern with only write accesses; it is also a worst-case pattern for our demand predictor. For the $\mathrm{AB}^{*}$ pattern the predictor will always make the wrong call: it will predict writes to $\mathrm{A}$ when the workload starts writing to $B$ and vice versa when the writes switch back to A. The usage distribution of Ouroboros for the $A^{*}$ benchmark is shown in Figure $7(b)$. The distribution can also be compared with those of DUSS and DDSS in Figure 1(e) and Figure 1(d), respectively, where all the writes were concentrated in one or two frames despite block migrations. However, the randomization introduced by Ouroboros elides the spikes created by deterministic swaps and distributes the writes over all the frames. Furthermore, as shown in Table 3, Ouroboros has significantly better $l_{2}$ and $l_{\infty}$ values than DUSS. In fact, DUSS itself shows very little benefit over no wear leveling in this case because it quickly gets stuck in the pattern where it is writing to only two 


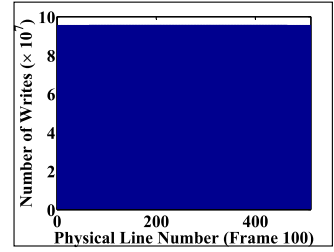

(a) $A^{*}$ pattern Frame 100 .

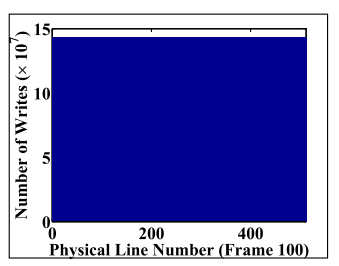

(b) AB* pattern Frame 100.

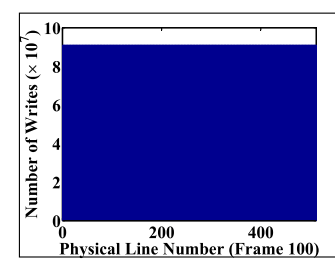

(c) $(\mathrm{AB}) * 50 \%$ pattern Frame 100 .

Fig. 8. Usage distribution within a frame for microbenchmark traces after local wear leveling.

frames (see Figure 1(e)). Ouroboros is comparable to randomized RUSS in this case since Ouroboros reverts to a random choice when its predictions are wrong.

The $(\mathrm{AB})^{*} \mathbf{5 0 \%}$ pattern is a workload that interpolates between the best- and worst-case patterns discussed earlier. It is a variant of the $\mathrm{AB}^{*}$ pattern that randomly chooses to write to block A or B in every epoch with equal probability. Hence, it makes a correct prediction for Ouroboros if the next block matches the current block and an incorrect prediction otherwise; that is, it has a $50 \%$ chance to make a correct prediction. The results for the $(\mathrm{AB})^{*} 50 \%$ benchmark are shown in Figure 7(c) and Table 3. As can be seen in the figure, even with $50 \%$ chance of correct prediction, Ouroboros achieves an extremely smooth usage distribution. The $l_{2}$ and $l_{\infty}$ metrics are also far superior to that of DUSS and RUSS. Hence, Ouroboros is able to use correct predictions to improve smoothness whenever possible.

From the microbenchmark experiments, we can conclude that Ouroboros has excellent behavior when the prediction is correct and is able to avoid continuous degradation if the predictions are incorrect. In cases (as would be the case in practice) when the predictions are partially correct, Ouroboros policies allow it to seamlessly exploit correct predictions to significantly improve wear leveling and to fall back to neutral, randomly chosen migration when incorrect, thereby achieving the best of both approaches.

Figure 8(a), Figure $8(\mathrm{~b})$, and Figure $8(\mathrm{c})$ shows the usage distribution within frame 100 for the three microbenchmarks. Since all three benchmarks only write to a single memory line in every global wear-leveling period, only one memory line will be written in this frame. The number of writes to that memory line is also the total number of writes to that frame. Therefore, without local wear leveling, the representative frame will have one heavily written memory line, while all the remaining lines will have no writes. With local wear leveling, the movement of the gap helps migrate the logical line to different physical memory lines within the frame in a round-robin manner; this allows the writes to be distributed smoothly across the whole frame. In this case, for all three benchmarks, the ratio of the usage between the highest- and lowest-usage memory lines in the frame is only around 1.001. Figure 8(a) clearly shows that local wear leveling can effectively distribute writes within a frame smoothly across its memory lines.

\section{Storage Benchmarks}

The MSR Cambridge storage trace [25] is a suite of block I/O traces of enterprise servers at Microsoft. For our experiment, a block request in each trace file is split into several L-byte (oneline) memory requests. The address of each request is recalculated according to its original block offset in the trace file and the offset of memory lines in that block. The requests of all the trace files are merged in the order of their original timestamps. We repeat the merged trace to obtain sufficient write requests to let the system finish 2,000 Ouroboros wear-leveling epochs. In this large trace, the number of read requests is around 2.36 times more than the number of write requests. 

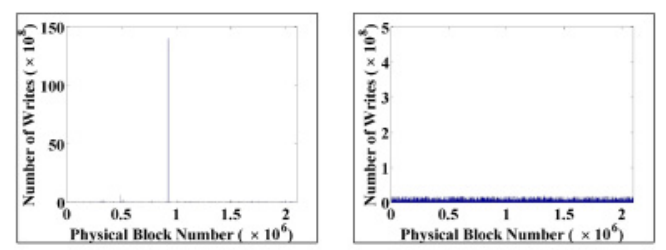

(a) MSR Cambridge without Wear-leveling. (b) MSR Cambridge after (c) Global Wear-leveling.

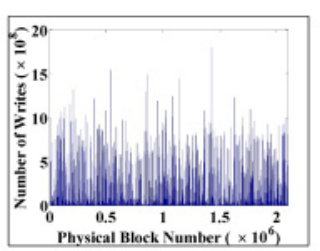

(c) FIU IODedup without Wear-leveling.

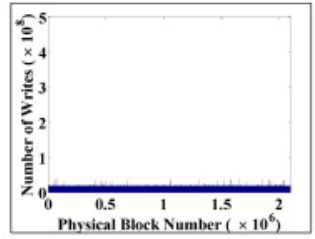

(d) FIU IODedup after Global Wear-leveling.

Fig. 9. Usage distribution across frames for storage benchmark traces with and without global wear leveling.

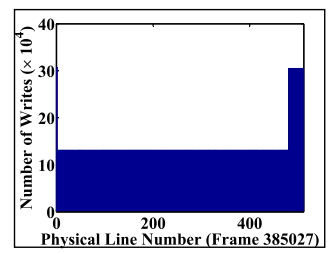

(a) MSR Cambridge (b) Frame 385027 without Fran Local Wear-leveling.
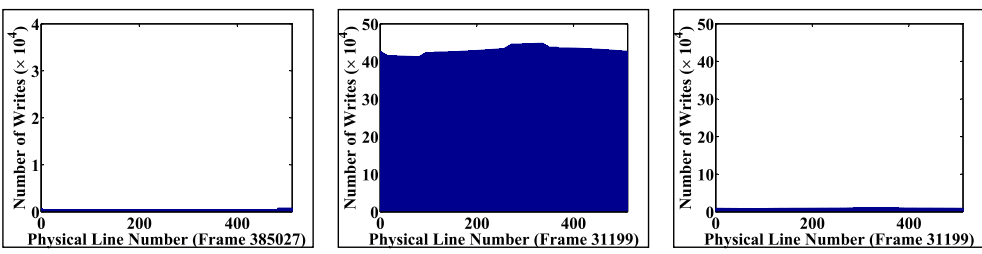

Fig. 10. Usage distribution within a frame for storage benchmark traces with and without local wear leveling.

Figure 9(a) shows the original write distribution of the MSR Cambridge benchmark. Based on the memory parameters selected in Table 2 , we have $2^{21}$ physical frames in these experiments. From the figure, we can see that the trace has several spikes with around the same number of writes. The frames with high spikes have $2 \times 10^{5}$ times the number of writes compared to the frames with nonzero low usage, which means that the writes are significantly nonuniform in this benchmark.

Figure 10(a) shows the original write distribution within frame number 385027 for the MSR Cambridge benchmark. We have 513 physical memory lines in each frame including the gap line. From the figure, we can see that there are a bunch of spikes in this frame when no local wear leveling is used. The highest-usage memory line has 2.35 times the number of writes compared to the lowest-usage memory line.

The FIU IODedup [16] traces are the activities collected by Florida International University on their storage servers. We do the same preprocessing for the FIU traces that we did for the MSR traces. In the combined trace, the number of write requests is around 3.97 times more than the number of read requests.

Figure 9(c) gives the original write distribution of the FIU IODedup benchmarks. Similar to the MSR Cambridge experiment, we have $2^{21}$ physical frames. From the figure, we can see that the heights of the spikes are more scattered than the MSR Cambridge benchmarks. The frames with high usage have around $1 \times 10^{7}$ times the number of writes compared to the low nonzero usage frames.

Figure 10(c) gives the original write distribution within frame number 31199 for the FIU IODedup traces. Similar to the MSR Cambridge experiment, we have 513 physical memory lines in each frame including the gap line. The usage distribution in this frame is less biased compared to the frame in the MSR Cambridge trace. The high-usage memory lines have 1.27 times the number of writes compared to the low-usage memory lines.

Figure 9(b) and Figure 9(d) show the usage distributions across frames of MSR Cambridge and FIU IODedup storage traces using Ouroboros wear leveling. The total number of writes to the 


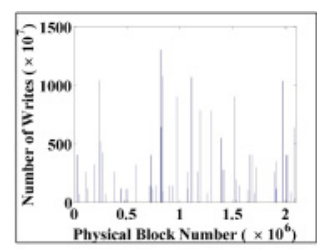

(a) gcc without Wearleveling.

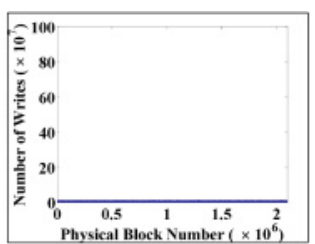

(b) gcc after Global Wear- ( leveling.
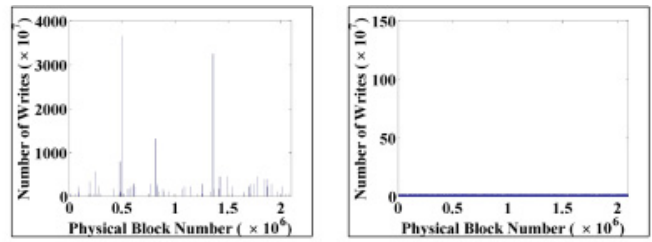

(c) mcf without Wear-(d) mcf after Global leveling. Wear-leveling.

Fig. 11. Usage distribution across frames for memory benchmark traces with and without global wear leveling.

memory is exactly the same as that without wear leveling. For MSR Cambridge storage traces, as can be seen in Figure 9(b), Ouroboros results in a roughly uniform number of $10^{7}$ writes per frame compared with the original, which had a two-level distribution of roughly $10^{10}$ writes per frame and $10^{5}$ writes per frame. Similarly, Figure 9(d) shows the usage distributions of FIU storage traces using Ouroboros wear leveling, which distributes the writes much more uniformly (order of $10^{7}$ ) than the distribution without wear leveling (order of $10^{9}$ for the high-usage frames).

Figure 10(b) and Figure 10(d) show the usage distributions within one frame for the MSR Cambridge and FIU IODedup storage traces, respectively, using local wear leveling. We choose the same frame number as the one used without wear leveling. For frame 385027 in the MSR Cambridge benchmarks, when local wear leveling is used, the high-usage memory lines have 1.74 times the number of writes of the low-usage memory lines. With local wear leveling, the writes in the frame are distributed more uniformly (order of $10^{2}$ ) than without local wear leveling (order of $10^{5}$ ). For frame number 31199 in the FIU IODedup benchmarks with local wear leveling, the high-usage memory lines have only 1.09 times the number of writes compared to the low-usage memory lines. Similar to the MSR results, usage for the frame with local wear leveling is of the order of $10^{3}$, which is much smoother compared to the frame without local wear leveling, whose usage is of the order of $10^{5}$. This also supports the conclusion we drew in the microbenchmark experiments that local wear leveling can effectively distribute the writes within frames smoothly across the memory lines.

\section{Memory Benchmarks}

The CPU SPEC 2006 [10] traces are a group of traces announced by the Standard Performance Evaluation Corporation (SPEC). They are benchmarks from real-life applications. Here, we use a subset of the 429.mcf and 403.gcc benchmarks for our memory analysis. Both mcf and gcc are memory-intensive benchmarks. The mcf benchmark is derived from MCF, a program used for single-depot vehicle scheduling in public mass transportation. In this trace, the number of read requests is around 1.439 times more than the number of write requests. The gcc benchmark is derived from the $\mathrm{C}$ language optimizing compiler. In this large trace, the number of read requests is around 2.08 times more than the number of write requests. We do the same preprocessing for the memory traces that we did for the storage traces.

Figure 11(a) and Figure 11(c) show the original interframe write distributions for the gcc and mcf traces, respectively. Similar to the storage experiments, we have $2^{21}$ physical blocks. The total number of writes for both the benchmarks are equal. From these figures, we can see that both traces have several spikes when no wear-leveling is used. From Table 4, we can see that in the case of no wear leveling, gcc has smaller $l_{2}$ and $l_{\infty}$ values. The write distribution of the gcc trace is smoother than the distribution of mcf.

Figure 12(a) and Figure 12(c) show the original intraframe write distributions for the gcc and mcf traces, respectively. As before, we have 513 physical memory lines in each frame including the 
Table 4. Storage and Memory Benchmark Measurements

\begin{tabular}{|l|l|l|l|l|l|l|l|l|}
\hline & \multicolumn{2}{|c|}{ MSR } & \multicolumn{2}{c|}{ FIU } & \multicolumn{2}{c|}{ gcc } & \multicolumn{2}{c|}{ mcf } \\
\hline $\begin{array}{l}\text { Wear-Leveling } \\
\text { Method }\end{array}$ & $\begin{array}{c}\boldsymbol{l}_{2} \\
\left(\times 10^{-6}\right)\end{array}$ & $\begin{array}{c}\boldsymbol{l}_{\infty} \\
\left(\times 10^{7}\right)\end{array}$ & $\begin{array}{c}\boldsymbol{l}_{2} \\
\left(\times 10^{-6}\right)\end{array}$ & $\begin{array}{c}\boldsymbol{l}_{\infty} \\
\left(\times 10^{7}\right)\end{array}$ & $\begin{array}{c}\boldsymbol{l}_{2} \\
\left(\times 10^{-6}\right)\end{array}$ & $\begin{array}{c}\boldsymbol{l}_{\infty} \\
\left(\times 10^{7}\right)\end{array}$ & $\begin{array}{c}\boldsymbol{l}_{2} \\
\left(\times 10^{-6}\right)\end{array}$ & $\begin{array}{c}\boldsymbol{l}_{\infty} \\
\left(\times 10^{7}\right)\end{array}$ \\
\hline \hline None & 24.33 & $1,399.5$ & 25.06 & 180.2 & 123.44 & $1,300.6$ & 186.27 & $3,648.50$ \\
\hline DUSS & 4.39 & 41.02 & 17.48 & 43.06 & 35.61 & 79.64 & 43.34 & 114.86 \\
\hline RUSS & 4.36 & 36.27 & 17.5 & 43.06 & 39.42 & 78.42 & 43.28 & 115.29 \\
\hline Ouroboros & 1.62 & 2.25 & 4.60 & 4.05 & 4.86 & 1.64 & 5.21 & 1.81 \\
\hline
\end{tabular}
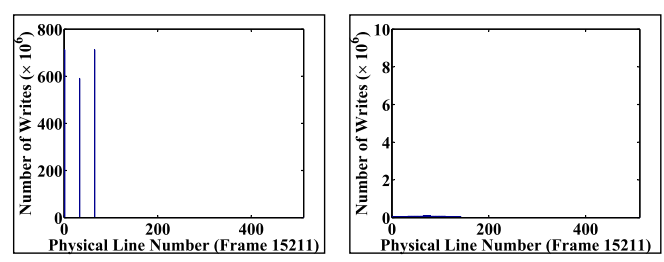

(a) gcc Frame 15211 with-(b) gcc Frame 15211 after (c) out Local Wear-leveling. Local Wear-leveling.
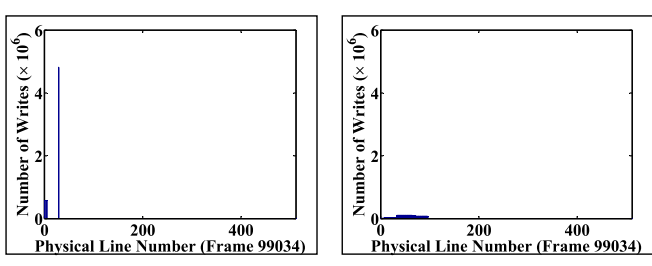

(c) mcf Frame 99034 with- (d) mcf Frame 99034 after out Local wear-leveling. Local Wear-leveling.

Fig. 12. Usage distribution within a frame for memory benchmark traces with and without global wear leveling.

gap line. In both traces, the writes are focused on a small number of memory lines in the frame, resulting in a highly skewed write distribution. The intraframe distribution is more skewed than that for the storage traces since the block writes in the latter cover several memory lines, while the memory accesses are sparse and more randomly distributed.

Figure 11(b) and Figure 11(d) show the interframe usage distributions for the gcc and mcf memory traces using Ouroboros wear leveling, respectively. The total number of writes to the memory is the same as without wear leveling. For the gcc trace, as can be seen in Figure 11(b), Ouroboros results in a far more uniform distribution; the high-usage frames have around $10^{6}$ writes per frame compared with the original, which has around $10^{10}$ writes. Similarly, Figure 11(d) shows the usage distribution for mcf using Ouroboros wear leveling; the number of writes of high-usage frames (order of $10^{6}$ ) is significantly smaller than that without wear leveling (order of $10^{10}$ ).

Figure 12(b) and Figure 12(d) show the intraframe usage distributions for gcc and mcf memory traces using local wear leveling, respectively. The total number of writes to the memory is the same as the experiments without wear leveling. For gcc with local wear leveling, representative frame 15211 has around $10^{5}$ writes per line, compared with $10^{8}$ without local wear leveling. For mcf the corresponding numbers for representative frame 99034 are $10^{5}$ with local wear leveling and $10^{6}$ without wear leveling.

From the usage distribution after wear leveling, we see that Ouroboros and local wear leveling result in the smoothest usage distribution for both storage and memory benchmarks compared to DUSS and RUSS. The smoothness metrics $l_{2}$ and $l_{\infty}$ in Table 4 and Figure 13 provide quantitative comparisons of Ourobroros against DUSS and RUSS for the storage and memory benchmarks. As can be seen, both the $l_{2}$ and $l_{\infty}$ metrics of Ouroboros are significantly better than that of the other two wear-leveling methods. Moreover, we notice that Ouroboros wear leveling only needs to do two block migrations every second with our experimental settings. However, the other two methods need to move 30 blocks every second and still have less even distributions. 


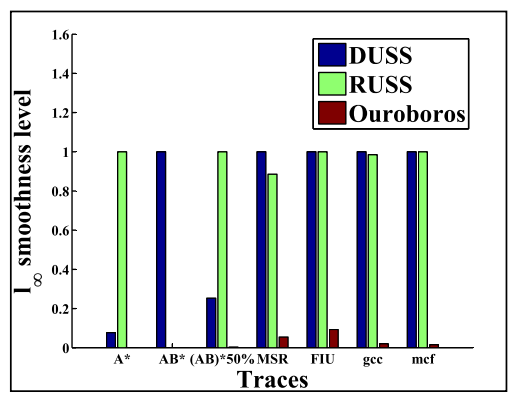

(a) $l_{\infty}$ smoothness level.

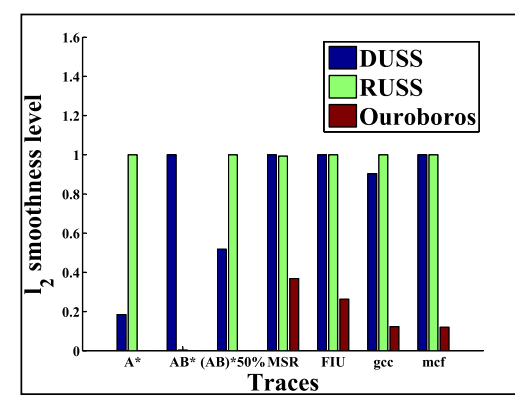

(b) $l_{2}$ smoothness level.

Fig. 13. Smoothness comparison among DUSS, RUSS, and Ouroboros wear leveling.

\subsection{Time Simulation Results}

In this section, we present simulation results on the time overhead of Ouroboros wear leveling. We simulate three benchmarks: microbenchmark $A^{*}$, the mfc memory benchmark, and the MSR Cambridge storage benchmark. For each benchmark we measured the number of cycles required to complete a given number of requests with and without wear leveling. For $\mathrm{A}^{*}, \mathrm{MSR}$, and $\mathrm{mfc}$ Cambridge experiments there are $2.99 \times 10^{8}, 3.59 \times 10^{8}$, and $3.2 \times 10^{8}$ requests, respectively, which includes sufficient writes to force several global wear-leveling events.

An event-driven simulator designed in accordance with the architecture described in Section 4 was developed. The controller inserts appropriate delays between requests to model the internal concurrency and latency of the NVRAM chips. We model four types of dependencies and three types of locality-based concurrency that affect the timing of requests. The four dependencies are write-to-write, write-to-read, read-to-write, and read-to-read. Locality-based concurrency refers to requests whose targets are to the same address, same partition, or different partitions. Different dependency and concurrency combinations result in different delay requirements between requests. For instance, the delay between two write requests is around 1,000 clock cycles, 100 clock cycles, and less than 10 clock cycles when the destination of two requests is to the same address, in the same partition, and in different partitions, respectively. The other types of dependencies will also slightly affect the delay times but to a lesser extent than the relative target addresses.

Local wear-leveling requests proceed concurrently with regular requests; internal interlocks are implemented to guard against races that may arise due to reordering of requests by the scheduler. For the global wear-leveling design, regular request processing is temporarily blocked during the block migration operations.

Figure 14(a) shows the simulation time for the $A^{*}$ microbenchmark. Surprisingly, the execution with wear leveling takes less time than the execution without wear leveling. Even though local wear leveling and global wear leveling cause additional reads and writes to the system, the experiment with these additional migration overheads runs a little faster than the experiments without the overheads. The reason is the long write-write delays necessary between writes to the same physical address. Following a local wear-leveling line migration, writes and reads of the new value can proceed concurrently with the pending writes to the old location. Moreover, since the $\mathrm{A}^{*}$ workload writes to only one frame during any epoch, global wear leveling requires only three block migrations, which is overshadowed by the decrease in the time caused by overlapping writes during local wear leveling. 


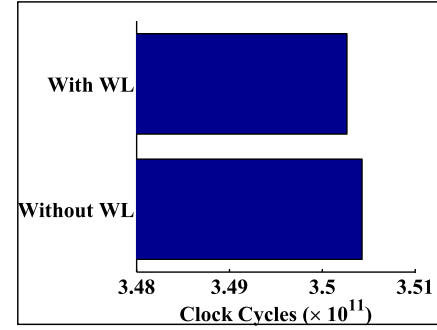

(a) A* Pattern after $2.99 \times 10^{8}$ regular requests.

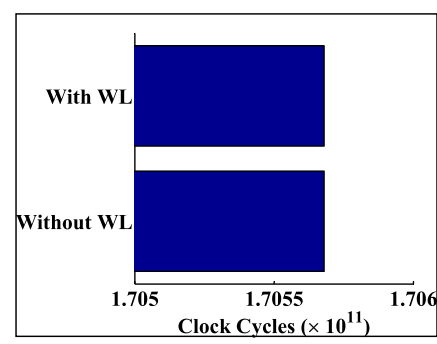

(b) MSR Cambridge after $3.59 \times$ $10^{8}$ regular requests.

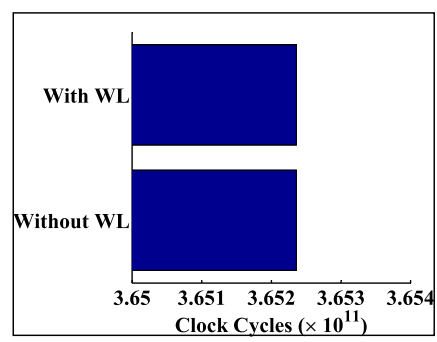

(c) mcf after $3.2 \times 10^{8}$ regular requests.

Fig. 14. Timeline for single record under $A^{*}$ Pattern, MSR Cambridge, and mcf.

Similar conclusions can be drawn for both of the other benchmarks. Figure 14(b) and Figure 14(c) show the execution times for the MSR Cambridge and mcf traces. In both cases, the increase in the execution time of an epoch is negligible due to the overlap with normal execution delays.

From the time analysis on micro-, storage, and memory benchmarks, we can see that in all of the cases, the local and global wear leveling only cause a small time overhead. In Section 6, we will estimate the theoretical upper-bound time overhead under worst-case assumptions; in all cases, the simulation results are within the estimated bounds.

\section{OVERHEAD EVALUATIONS AND PARAMETER SETTINGS}

In this section, the time and space overheads for wear leveling and the tradeoffs between them are modeled and analyzed. Moreover, we also discuss how the design parameters are chosen to meet specified smoothness and overhead constraints.

\subsection{Wear Leveling Constraints}

Ouroboros wear leveling must satisfy two endurance constraints called the local constraint (Equation (3)) and the global constraint (Equation (4)). The local constraint requires that the maximum number of writes to one memory line in any frame before it relocates by the local wear leveling to be less than a parameter $\tau$. The worst-case number of writes to a memory line between successive relocations by local wear leveling is $(F / L) \cdot \Gamma_{L}$, where $F$ is the frame size, $L$ is the line size, and $\Gamma_{L}$ is the local reorganization threshold. The local constraint (Equation (3)) limits this to be $\tau$. The global wear-leveling constraint requires that the expected number of writes to a memory line between relocations by global wear leveling is also bounded by $\tau$. The maximum number of writes to a frame between successive global wear-leveling events is $\Gamma_{G}$. The average number of writes to any line within the frame is $\Gamma_{G} /(F / L)$. The global constraint (Equation (4)) limits this to be $\tau$.

$$
\begin{aligned}
& f\left(F, \Gamma_{L}\right)=\frac{F \cdot \Gamma_{L}}{L} \leq \tau \\
& g\left(F, \Gamma_{G}\right)=\frac{\Gamma_{G} \cdot L}{F} \leq \tau
\end{aligned}
$$

\subsection{Time Overhead}

In this section, we will analyze time overhead in two respects. The first part of time overhead is the overhead of one additional level of addressing involved in every request. The second part of the time overhead is the processing time for additional local and global wear leveling. 
The memory access time is composed of two parts (Equation (5)). The first part $T_{V A->L A}$ is the time for the processor MMU to translate the virtual address (VA) into the NVRAM logical address (LA). This part is the same as the addressing part without wear leveling in the traditional memory system. In the traditional memory system, the LA here is the machine address to the memory devices. The time overhead involved by wear leveling is the second part in Equation (5) that is incurred in the NVRAM controller to translate the LA into the NVRAM PA (Equation (6)). As is shown in Equation (7), the translation of the LBN to the PFN involves looking up the frame table. Since parts of the frame table can be cached in SRAM, the time taken depends on the hit ratio of the SRAM cache and the speeds of SRAM and NVRAM read accesses. The $T_{L A->P A}$ also involves performing the offset translation for local wear leveling; since the reads of the relevant counters and gap and start pointers are done concurrently with the frame table access, this adds negligible additional overhead.

$$
\begin{gathered}
T_{\text {addressing }}=T_{V A->L A}+T_{L A->P A} \\
T_{L A->P A}=\max \left(T_{L B N->P F N}, T_{L W L \_ \text {addressing }}\right) \\
T_{L B N->P F N}=\left\{\begin{array}{l}
T_{S R A M \_R E A D} \text { FT Hit } \\
T_{N V R A M \_R E A D} \text { FT Miss }
\end{array}\right.
\end{gathered}
$$

The second part of the time overhead is created by the additional requests that need to be processed to realize local GapLine movement and global block migration for local and global wear leveling separately.

The time overhead can be calculated by multiplying the total number of local wear-leveling operations done in $n$ global wear-leveling periods by the time for each migration. An upper bound on the total time spent on local wear-level migrations in $n$ epochs is shown in Equation (8). This follows because there are $\Gamma_{G}$ writes in each epoch and local wear-leveling migrations in a frame must be at least $\Gamma_{L}$ writes apart. The copying of a line is assumed to take time $T_{r w}$. The overestimate in the upper bound arises because local wear-leveling operations in different frames can be closely spaced depending on the distribution of writes. However, the error term is bounded by $N \times(1-$ $\frac{1}{\Gamma_{L}}$ ), a fixed quantity that becomes negligible as the number of epochs $n$ increases.

$$
t_{L W L} \leq \frac{n \cdot \Gamma_{G} \cdot T_{r w}}{\Gamma_{L}}
$$

A global wear-leveling migration mainly requires relocating a maximum of $N_{m}$ blocks in any epoch. The global wear-leveling time overhead $t_{G W L}$ for $n$ epochs is shown in Equation (9). With a frame size of $F$ bytes and a memory line size of $L$ bytes, the number of lines to be copied per block is $F / L$ with a time overhead of $T_{r w}$ per line.

The time required for $n$ epochs at a write rate of $v / L$ writes/sec ( $v$ bytes/sec) is given in Equation (10). The time overhead is defined as the ratio of the time spent on wear-leveling migrations to the time for actual writes and is given in Equation (11). According to Equation (11), there are three variables, which are $F, \Gamma_{L}$, and $\Gamma_{G}$. Increasing $F$ leads to an increase in the percentage time overheads, but increasing $\Gamma_{L}$ and $\Gamma_{G}$ lead to a decrease in the percentage timing overhead. We can get the theoretical time overhead from Equation (11). The time overheads are $0.63 \%, 0.52 \%$, and $0.52 \%$ for the microbenchmarks, storage workloads, and memory workloads, respectively. In Section 5.4, we did the time simulation experiments on micro, storage, and memory workloads. The results also show that in the storage and memory workloads, when request order optimizations 
are involved, the time overheads caused by the wear leveling will be much smaller than the value calculated by theoretical analysis without any optimization.

$$
\begin{gathered}
t_{G W L}=\frac{n \cdot N_{m} \cdot F \cdot T_{r w}}{L} \\
T_{\text {total }}=\frac{n \cdot \Gamma_{G} \cdot L}{v} \\
p_{t}\left(F, \Gamma_{G}, \Gamma_{L}\right)=\frac{t_{L W L}+t_{G W L}}{T_{\text {total }}} \leq\left(\frac{1}{\Gamma_{L}}+\frac{N_{m} \cdot F}{\Gamma_{G} \cdot L}\right) \frac{T_{r w} \cdot v}{L}
\end{gathered}
$$

\subsection{Space Overhead}

The memory is partitioned across $C$ chips and requests are always striped across all chips. We concentrate on a single chip of size $M$ bytes that has frames of size $F$ and a line of size $L$. The number of frame descriptors required in the frame table is $M / F$. One frame table is sufficient for all chips since they will be relocated as a complete stripe. With $S_{D}$ bytes required per descriptor, the total space for the global wear leveling is given by Equation (12). The space overhead for local wear leveling is dominated by the space for the GapLines in each frame. Equation (13) gives the space overhead for local wear leveling in all $C$ chips.

As is shown in Equation (14), space overhead decreases with increasing $F$, since both the frame table size and the number of GapLines decrease. According to the equation, we can see that there is only one variable $\mathrm{F}$ in the space overhead equation. It means that the percentage space overhead is only related to the frame size. In our experiments with our selected parameters, the space overheads are $0.2 \%$ for the microbenchmark and storage and memory workloads.

$$
\begin{gathered}
S_{G W L}=\frac{S_{D} \cdot M}{F} \\
S_{L W L}=\frac{L \cdot M \cdot C}{F} \\
p_{S}(F)=\frac{S_{L W L}+S_{G W L}}{C \cdot M}=\frac{S_{D}+L \cdot C}{F \cdot C}
\end{gathered}
$$

\subsection{Write Amplification Analysis}

Additional writes caused by global wear leveling and local wear leveling lead to write amplification. Actual writes to the memory are a multiple of the intended writes to it. Therefore, write amplification can be calculated by dividing the actual number of memory write requests in $n$ epochs by the number of effective memory write requests in the same period. The total number of effective write requests during $n$ epochs is the number of writes $n \cdot \Gamma_{G}$. For global wear leveling, the number of additional write requests is given by Equation (15). $R_{G W L}$ represents the total number of additional block migration requests needed in $n$ epochs. Equation (16) represents the total number of additional line migration requests needed in $n$ epochs. Equation (17) represents the write amplification. According to the equation, $A_{u}$ depends on three variables, $F, \Gamma_{G}$, and $\Gamma_{L}$. The increase of $F$ causes the write amplification to increase, and the increase of $\Gamma_{G}$ and $\Gamma_{L}$ leads to a decrease in the write amplification. In our experiments with our selected parameters, the write amplification is $1.0053,1.0067$, and 1.0067 for the microbenchmarks and storage and memory workloads, respectively. The write amplification trend, not surprisingly, follows that of the timing overhead, 


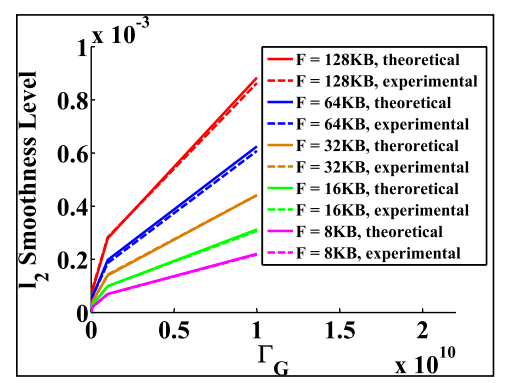

Fig. 15. Relationship among $l_{2}$ smoothness level, global wear-leveling threshold $\left(\Gamma_{G}\right)$, and block size $(F)$.

and the magnitude of write amplification is quite small. Therefore, in Section 6.6, we only take timing overhead into consideration.

$$
\begin{gathered}
R_{G W L}=\frac{n \cdot N_{m} \cdot F}{L} \\
R_{L W L}=\frac{n \cdot \Gamma_{G}}{\Gamma_{L}} \\
A_{u}\left(F, \Gamma_{G}, \Gamma_{L}\right)=\frac{n \cdot \Gamma_{G}+R_{G W L}+R_{L W L}}{n \cdot \Gamma_{G}}=1+\frac{1}{\Gamma_{L}}+\frac{N_{m} \cdot F}{\Gamma_{G} \cdot L}
\end{gathered}
$$

\subsection{Upper Bound on $\boldsymbol{l}_{2}$ Smoothness}

We now model the relationship between the $l_{2}$ smoothness, the granularity of global wear leveling $F$, and the threshold of global wear leveling $\Gamma_{G}$. We use a reference benchmark made up of $W$ writes to randomly selected blocks; each block is written $\Gamma_{G}$ times consecutively before the next block is selected. The statistics from this model closely match ${ }^{3}$ that of the $\mathrm{AB}^{*}$ reference pattern, which is a worst-case sequence for Ouroboros.

The distribution of frames written to by the $\mathrm{AB}^{*}$ reference pattern is similar to the distribution of balls in the balls-and-bins problem. For the $\mathrm{AB}^{*}$ reference pattern doing $W$ memory writes is similar to throwing $\frac{W}{\Gamma_{G}}$ balls into $N-1$ bins. Since we know that our prediction is always incorrect, the next frame to be written will always be a randomly selected block from the $N-1$ blocks that exclude the lowest-usage frame; the lowest-usage frame will hold the wrongly predicted block after migration. For the balls-and-bins problem, the random variable $X$ represents the number of balls in the same bin. $X$ obeys binomial distribution with mean $\mu=n \cdot p$ and standard deviation $\sigma=\sqrt{n \cdot p \cdot(1-p)}$, where $n$ represents the number of balls and $p$ represents the probability for a ball falling in a bin. This means $n=\frac{W}{\Gamma_{G}}$ and $p=\frac{1}{N-1}$ in the $\mathrm{AB}^{*}$ reference pattern. Since each ball represents $\Gamma_{G}$ writes, the standard deviation of the usage distribution $\left(\sigma_{W L}\right)$ equals the standard deviation of the random variable $U=\Gamma_{G} \cdot X$ and can be calculated through Equation (18). $\sigma_{W L}$ is also the $l_{2}$ smoothness parameter for the $\mathrm{AB}^{*}$ reference pattern.

Figure 15 shows a plot of the $l_{2}$ parameter versus $\Gamma_{G}$ for different frame sizes. The dotted lines figure is generated empirically by experiments on the reference benchmark with about $2.7 \times 10^{12}$ writes ( 1 day of continuous writes). The full lines represent the theoretical value calculated through Equation (18). This figure is consistent with our theoretical $l_{2}$ smoothness equation. Given a

\footnotetext{
${ }^{3}$ Ouroboros will randomly select from N - 1 frames, while the reference benchmark will select from N frames. For large $\mathrm{N}$ the difference is negligible.
} 
Table 5. Parameters in the Parameter Selection System

\begin{tabular}{|l|c|l|c|l|c|}
\hline \multicolumn{2}{|c|}{ Input } & \multicolumn{2}{c|}{ Output } & \multicolumn{2}{c|}{ System Parameters } \\
\hline Parameter & Symbol & \multicolumn{1}{|c|}{ Parameter } & Symbol & \multicolumn{1}{|c|}{ Parameter } & Symbol \\
\hline \hline Endurance Limit & $\tau$ & Frame Size & $\mathrm{F}$ & Memory Size & $\mathrm{M}$ \\
\hline Time Limit & $\Omega_{t}$ & Global Threshold & $\Gamma_{G}$ & Striping Level & $\mathrm{C}$ \\
\hline Space Limit & $\Omega_{s}$ & Local Threshold & $\Gamma_{L}$ & Memory Line Size & $\mathrm{L}$ \\
\hline Smoothness Limit & $l_{2}$ & & & Write Rate & $v$ \\
\hline & & & & Read-to-Write Time & $T_{r w}$ \\
\hline & & & & Max Blocks Migrated & $N_{m}$ \\
\hline
\end{tabular}

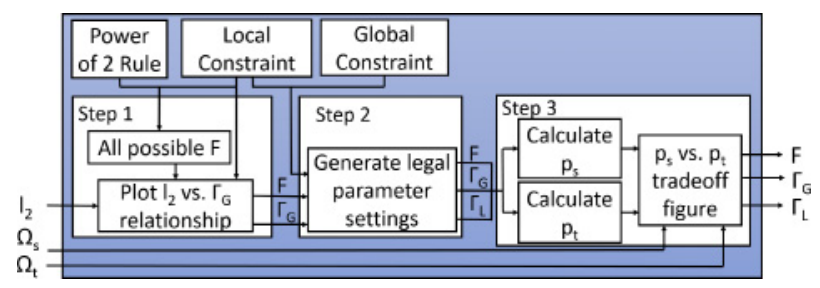

Fig. 16. Flowchart for parameter selection.

threshold $l_{2}$ level as a design constraint, the graph indicates feasible ranges of $\left(F, \Gamma_{G}\right)$ values, which will be used in the design procedure described in Section 6.6.

$$
\begin{aligned}
\sigma_{W L}\left(F, \Gamma_{G}\right) & =\sqrt{\operatorname{Var}(U)} \\
& =\sqrt{\operatorname{Var}\left(\Gamma_{G} \cdot X\right)} \\
& =\Gamma_{G} \cdot \sigma \\
& =\Gamma_{G} \cdot \sqrt{n \cdot p \cdot(1-p)} \\
& =\Gamma_{G} \cdot \sqrt{\frac{W}{\Gamma_{G}} \cdot \frac{1}{N-1}\left(1-\frac{1}{N-1}\right)} \\
& =\sqrt{\Gamma_{G} \cdot W \cdot\left(\frac{F}{M-F}-\left(\frac{F}{M-F}\right)^{2}\right)}
\end{aligned}
$$

\subsection{Optimizing Parameters Based on Overhead Bounds}

Three steps to select suitable values for the wear-leveling parameters are discussed in this section. As shown in Table 5, the three output design parameters we need are frame size $(\mathrm{F})$ (which is also the unit size for global wear leveling) and global threshold $\left(\Gamma_{G}\right)$ and local threshold $\left(\Gamma_{L}\right)$ (which reflect the frequency of global and local wear-leveling operations, respectively). The four input parameters in Table 5 constrain the design. These are the endurance limit $(\tau)$, which is used in Equation (3) and Equation (4); time and space overhead limits $\left(\Omega_{t}\right.$ and $\Omega_{s}$ ), which use constraints (Equation (11) and Equation (14)) to limit the time and space overhead; and smoothness limit $l_{2}$, which determines the frequency of wear leveling needed to meet the specified limit. In addition, three fixed system parameters are also inputs to the design; these are memory size $M$, striping Level $C$, and memory line size $L$. Note that the size of a memory request is $C \times L$ bytes, that is, a stripe over $C$ chips of one memory line per chip.

The flowchart in Figure 16 shows the three steps in selecting the parameters. First, using the endurance limit $\tau$ and the local constraint requirement in Equation (3), we find an upper bound of 


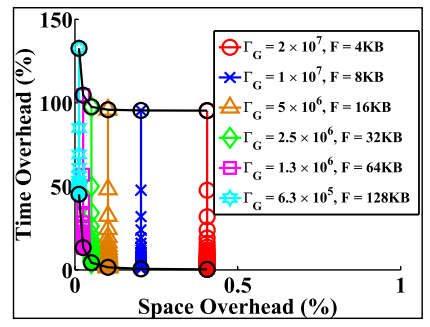

Fig. 17. Tradeoff between time and space overhead when $l_{2}=7 \times 10^{-6}$.

$\tau / \Gamma_{L}$ for $F / L$, which is the number of memory lines in a block. Since $\Gamma_{L} \geq 1$, we have $1 \leq F / L \leq \tau$. Since both the frame and line sizes are powers of $2, F / L$ needs to be a power of 2 . Therefore, there are only a limited number (at $\operatorname{most} \log _{2} \tau+1$ ) of feasible values for $F / L$ to consider. Furthermore, since $L$ is set by the memory system design, this also fixes the number of feasible $F$ values. For each $F$ we can upper bound $\Gamma_{G}$ using Equation (4).

As a running example, suppose $\tau=1 \times 10^{5}$, which is $1 \%$ of a memory cell's lifetime. This leads to the requirement: $1 \leq F / L \leq \tau \approx 2^{17}$. Since $F$ and $L$ must be powers of 2 , we have only 18 different choices for the frame size. Since $L$ is set by the memory system design at 16 bytes (based on the request and stripe sizes), the frame size $F$ must be a power of 2 between 16 bytes and $2 \mathrm{MB}$. For each feasible $F$ and the fixed value of $L$, we can find an upper bound on the value of $\Gamma_{G}$ equal to $\tau \cdot F / L$ from Equation (4).

Next, for each value of $F$ we find the maximum value of $\Gamma_{G}$ that meets a given smoothness limit $l_{2}$. We use the smoothness curves of Figure 15 for this purpose. For any $F$, this is the intersection of the horizontal line representing the target $l_{2}$ value with the smoothness curve for that $F$. If the corresponding $\Gamma_{G}$ exceeds the upper bound from Equation (4) mentioned earlier, then we set $\Gamma_{G}$ at the upper bound. While any smaller value of $\Gamma_{G}$ will also satisfy the smoothness limit, it only makes the time overhead larger (Equation (11)), and hence they are not considered going forward.

At this point we have a set of feasible values for $F$, and for each such value the best choice of $\Gamma_{G}$. Next we determine feasible values for $\Gamma_{L}$ for each $F$ using the local constraint in Equation (3).

The third step is to calculate the time and space overhead for each possible combination of $F$, $\Gamma_{G}$, and $\Gamma_{L}$ and get a tradeoff curve like the one in Figure 17. Points in Figure 17 represents legal parameter settings for the selected $l_{2}$ smoothness level. After we get the time and space tradeoff plot, we can choose an optimal point in Figure 17 that satisfies the time and space overhead limits $\Omega_{t}$ and $\Omega_{s}$. Different boundary points represent different feasible time and space overhead settings. The designer can weight the importance of the space and time overheads appropriately in making a final choice.

Table 6 gives an example of selecting parameters for the $512 \mathrm{MB}$ memory (microbenchmark) when $l_{2}=7 \times 10^{-6}$. The 10 parameter settings listed in the table all meet local and global constraints. The time overhead grows sharply with larger $F$. Moreover, the tradeoff between space and time overheads can also be noticed in Figure 17.

The three-step method to select optimized wear-leveling parameters can also be modeled as an optimization problem in Equation (19). The fourth and fifth constraints in the optimization equation are our local wear-leveling constraint (Equation (3)) and global wear-leveling constraint (Equation (4)), respectively. Our target is to find optimized parameter settings for $F, \Gamma_{G}$, and $\Gamma_{L}$ that minimize both the time and space overhead and keep a balance between them under certain constraints. By using all the constraints, we can simplify the problem to finding optimized $F$ instead of three variables. Other variables $\Gamma_{G}$ and $\Gamma_{L}$ can be generated from the listed constraints in 
Table 6. Legal Parameter Settings When $L_{2}=7 \times 10^{-6}$

\begin{tabular}{|c|l|l|l|l|l|}
\hline Options & \multicolumn{1}{|c|}{$F$} & $\Gamma_{G}$ & $\Gamma_{L}$ & \multicolumn{1}{|c|}{$\Omega_{s}(\%)$} & \multicolumn{1}{|c|}{$\Omega_{t}(\%)$} \\
\hline \hline 1 & $2 \mathrm{MB}$ & $4 \times 10^{4}$ & 1 & $7.9 \times 10^{-4}$ & $9,452.94$ \\
\hline 2 & $1 \mathrm{MB}$ & $7.9 \times 10^{4}$ & 2 & $1.6 \times 10^{-3}$ & $2,425.19$ \\
\hline 3 & $512 \mathrm{~KB}$ & $1.6 \times 10^{5}$ & 3 & $3.2 \times 10^{-3}$ & 628.17 \\
\hline 4 & $256 \mathrm{~KB}$ & $3.1 \times 10^{5}$ & 6 & $6.3 \times 10^{-3}$ & 165.1 \\
\hline 5 & $128 \mathrm{~KB}$ & $6.3 \times 10^{5}$ & 12 & $1.3 \times 10^{-2}$ & 45.25 \\
\hline 6 & $64 \mathrm{~KB}$ & $1.3 \times 10^{6}$ & 24 & $2.5 \times 10^{-2}$ & 13.3 \\
\hline 7 & $32 \mathrm{~KB}$ & $2.5 \times 10^{6}$ & 48 & $5.1 \times 10^{-2}$ & 4.28 \\
\hline 8 & $16 \mathrm{~KB}$ & $5 \times 10^{6}$ & 97 & 0.1 & 1.55 \\
\hline 9 & $8 \mathrm{~KB}$ & $1 \times 10^{7}$ & 195 & 0.2 & 0.63 \\
\hline 10 & $4 \mathrm{~KB}$ & $2 \times 10^{7}$ & 391 & 0.4 & 0.28 \\
\hline
\end{tabular}

the optimization equation. The theoretical $l_{2}$ smoothness (STD) in Equation (18) can be simplified to Equation (20). By transforming Equation (20) together with the first constraint in Equation (19), we can get Equation (21). Then, by transforming our local wear-leveling constraint, we can get Equation (22). Finally, by combining Equation (21) and Equation (22), we can generate a new version of time overhead constraint in optimization equation as is shown in Equation (23). Because we want the $p_{t}$ to be as small as possible, the smallest value $p_{t}$ can be generated from the equation in the first line of Equation (23), and this equation is only related to $F$. Moreover, $p_{s}$ is also only related to $F$ and can be simplified from Equation (14) to Equation (24). As discussed earlier in this section, there are only a logarithmic number of choices for the frame size $\mathrm{F}$. Therefore, we can get no more than $\left(\log _{2} \tau+1\right)$ feasible pairs of values for the time and space overheads. These are the bottom most points of each vertical line in Figure 17. The value pairs which are theoretically calculated from our optimization problem match the experimental values closely.

$$
\begin{array}{cl}
\min _{F, \Gamma_{G}, \Gamma_{L}} & \alpha_{1} \cdot \Omega_{t}\left(F, \Gamma_{G}, \Gamma_{L}\right)+\alpha_{2} \cdot \Omega_{s}(F) \\
\text { subject to } \quad & \sigma_{W L}\left(F, \Gamma_{G}\right) \leq l_{2} \\
& p_{t}\left(F, \Gamma_{G}, \Gamma_{L}\right) \leq \Omega_{t} \\
& p_{s}(F) \leq \Omega_{s} \\
& f\left(F, \Gamma_{L}\right) \leq \tau \\
& g\left(F, \Gamma_{G}\right) \leq \tau \\
\sigma_{W L}=\sqrt{\Gamma_{G} \cdot W \cdot\left(\frac{F}{M-F}-\left(\frac{F}{M-F}\right)^{2}\right)} \leq l_{2} \\
\frac{F}{\Gamma_{G}} \geq \frac{F \cdot\left(\frac{F}{M-F}-\left(\frac{F}{M-F}\right)^{2}\right)}{l_{2}^{2}} \\
\frac{1}{\Gamma_{L}} \geq \frac{F}{\tau \cdot L}
\end{array}
$$




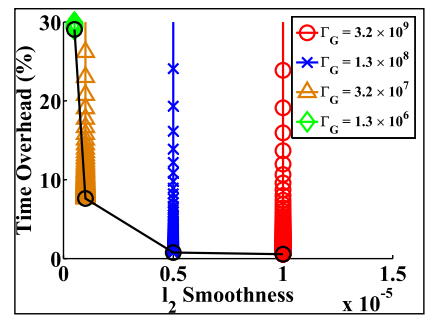

Fig. 18. Tradeoff between time overhead and $l_{2}$ smoothness when $F=8 \mathrm{~KB}$.

$$
\begin{aligned}
& \frac{T_{r w} \cdot v}{L} \cdot\left(\frac{F}{\tau \cdot L}+\frac{N_{m} \cdot F \cdot W \cdot\left(\frac{F}{M-F}-\left(\frac{F}{M-F}\right)^{2}\right)}{L \cdot l_{2}^{2}}\right) \\
& \quad \leq p_{t}=\left(\frac{1}{\Gamma_{L}}+\frac{N_{m} \cdot F}{\Gamma_{G} \cdot L}\right) \frac{T_{r w} \cdot v}{L} \\
& \leq \Omega_{t}
\end{aligned}
$$

To see the tradeoff between $l_{2}$ smoothness level and time overhead, we generated Figure 18 . We set $F$ to be $8 \mathrm{~KB}$, which also fixes the space overhead. Then we select four different $l_{2}$ smoothness levels and examine their time overhead for different $\Gamma_{G}$ and $\Gamma_{L}$. The points in the same vertical line have same $\Gamma_{G}$ and $l_{2}$ smoothness value. The bottommost point in each vertical line represents the smallest time overhead at that smoothness level.

\section{CONCLUSION}

In this article, we proposed a novel hierarchical wear-leveling model called Ouroboros wear leveling. Ouroboros uses a two-level strategy that combines frequent low-cost intraregion wear leveling with interregion wear leveling at a larger time interval and spatial granularity. Ouroboros is a hybrid migration scheme that exploits correct demand predictions in making better wear-leveling decisions while using randomization to avoid worst-case destructive write patterns. In contrast, existing schemes are either random or deterministic; in the first case, they fail to exploit knowledge of access patterns to do better than average, while deterministic strategies are vulnerable to malicious attacks. We also described a systematic method to choose the wear-leveling parameters (frame size, line size, and frequency of local and global migrations) while meeting constraints on endurance, smoothness, and time and space overheads. The system was evaluated using simulation on synthetic microbenchmarks, two standard storage traces, and two standard memory traces. The evaluation demonstrates that it is possible to keep the writes smoothly distributed across the memory with no more than $0.2 \%$ space overhead and $0.52 \%$ time overhead for a $512 \mathrm{~GB}$ memory and a $(32 \times 500) \mathrm{MB} / \mathrm{s}$ write rate.

\section{REFERENCES}

[1] Yoshihiro Arimoto and Hiroshi Ishiwara. 2004. Current status of ferroelectric random-access memory. Mrs Bulletin 29, 11 (2004), 823-828.

[2] Amir Ban. 2004. Wear leveling of static areas in flash memory. (May 4, 2004). Retrieved from https://www.google. com/patents/US6732221. US Patent 6,732,221.

[3] Avraham Ben-Aroya and Sivan Toledo. 2006. Competitive analysis of flash-memory algorithms. In European Symposium on Algorithms. Springer, 100-111. 
[4] Geoffrey W. Burr, Matthew J. Breitwisch, Michele Franceschini, Davide Garetto, Kailash Gopalakrishnan, Bryan Jackson, Bülent Kurdi, Chung Lam, Luis A. Lastras, Alvaro Padilla, Bipin Rajendran, Simone Raoux, and Rohit S. Shenoy. 2010. Phase change memory technology. Journal of Vacuum Science \& Technology B, Nanotechnology and Microelectronics: Materials, Processing, Measurement, and Phenomena 28, 2 (2010), 223-262.

[5] Li-Pin Chang. 2007. On efficient wear leveling for large-scale flash-memory storage systems. In Proceedings of the 2007 ACM Symposium on Applied Computing. ACM, 1126-1130.

[6] Chieh-Fang Chen, A. Schrott, M. H. Lee, S. Raoux, Y. H. Shih, M. Breitwisch, F. H. Baumann, E. K. Lai, T. M. Shaw, P. Flaitz, R. Cheek, E. A. Joseph, S. H. Chen, B. Rajendran, H. L. Lung, and C. Lam. 2009. Endurance improvement of Ge2Sb2Te5-based phase change memory. In 2009 IEEE International Memory Workshop (IMW'09). IEEE, 1-2.

[7] Sangyeun Cho and Hyunjin Lee. 2009. Flip-N-Write: A simple deterministic technique to improve PRAM write performance, energy and endurance. In 42nd Annual IEEE/ACM International Symposium on Microarchitecture, 2009 (MICRO42). IEEE, 347-357.

[8] Xiangyu Dong, Xiaoxia Wu, Guangyu Sun, Yuan Xie, Helen Li, and Yiran Chen. 2008. Circuit and microarchitecture evaluation of 3D stacking magnetic RAM (MRAM) as a universal memory replacement. In 45th ACM/IEEE Design Automation Conference, 2008 (DAC'08). IEEE, 554-559.

[9] Alexandre P. Ferreira, Miao Zhou, Santiago Bock, Bruce Childers, Rami Melhem, and Daniel Mossé. 2010. Increasing PCM main memory lifetime. In Proceedings of the Conference on Design, Automation and Test in Europe. European Design and Automation Association, 914-919.

[10] John L. Henning. 2006. SPEC CPU2006 benchmark descriptions. ACM SIGARCH Computer Architecture News 34, 4 (2006), 1-17.

[11] Jingtong Hu, Qingfeng Zhuge, Chun Jason Xue, Wei-Che Tseng, and Edwin H.-M. Sha. 2013. Software enabled wearleveling for hybrid PCM main memory on embedded systems. In 2013 Design, Automation \& Test in Europe Conference \& Exhibition (DATE'13). IEEE, 599-602.

[12] Yiming Huai. 2008. Spin-transfer torque MRAM (STT-MRAM): Challenges and prospects. AAPPS Bulletin 18, 6 (2008), 33-40.

[13] Corporation Intel. 2016. Persistent memory programming. Retrieved from http://pmem.io.

[14] Engin Ipek, Jeremy Condit, Edmund B. Nightingale, Doug Burger, and Thomas Moscibroda. 2010. Dynamically replicated memory: Building reliable systems from nanoscale resistive memories. In ACM SIGARCH Computer Architecture News, Vol. 38. ACM, 3-14.

[15] Yongsoo Joo, Dimin Niu, Xiangyu Dong, Guangyu Sun, Naehyuck Chang, and Yuan Xie. 2010. Energy-and enduranceaware design of phase change memory caches. In Proceedings of the Conference on Design, Automation and Test in Europe. European Design and Automation Association, 136-141.

[16] Ricardo Koller and Raju Rangaswami. 2010. I/O deduplication: Utilizing content similarity to improve I/O performance. ACM Transactions on Storage (TOS) 6, 3 (2010), 13.

[17] Emre Kültürsay, Mahmut Kandemir, Anand Sivasubramaniam, and Onur Mutlu. 2013. Evaluating STT-RAM as an energy-efficient main memory alternative. In 2013 IEEE International Symposium on Performance Analysis of Systems and Software (ISPASS'13). IEEE, 256-267.

[18] Benjamin C. Lee, Engin Ipek, Onur Mutlu, and D. B. 2009. Architecting phase change memory as a scalable dram alternative. In ACM SIGARCH Computer Architecture News 37, 3 (2009), 2-13.

[19] Benjamin C. Lee, Engin Ipek, Onur Mutlu, and Doug Burger. 2010. Phase change memory architecture and the quest for scalability. Communications of the ACM 53, 7 (2010), 99-106.

[20] Benjamin C. Lee, Ping Zhou, Jun Yang, Youtao Zhang, Bo Zhao, Engin Ipek, Onur Mutlu, and Doug Burger. 2010. Phase-change technology and the future of main memory. IEEE Micro 30, 1 (2010), 143.

[21] Dean L. Lewis and Hsien-Hsin S. Lee. 2009. Architectural evaluation of 3D stacked RRAM caches. In IEEE International Conference on 3D System Integration (3DIC). IEEE, 1-4.

[22] Duo Liu, Tianzheng Wang, Yi Wang, Zili Shao, Qingfeng Zhuge, and Edwin Sha. 2013. Curling-PCM: Applicationspecific wear leveling for phase change memory based embedded systems. In 2013 18th Asia and South Pacific Design Automation Conference (ASP-DAC'13). IEEE, 279-284.

[23] Jagan Singh Meena, Simon Min Sze, Umesh Chand, and Tseung-Yuen Tseng. 2014. Overview of emerging nonvolatile memory technologies. Nanoscale Research Letters 9, 1 (2014), 1-33.

[24] Sparsh Mittal and Jeffrey S. Vetter. 2015. AYUSH: A technique for extending lifetime of SRAM-NVM hybrid caches. In IEEE Computer Architecture Letters 14, 2 (2015), 115-118.

[25] Dushyanth Narayanan, Austin Donnelly, and Antony Rowstron. 2008. Write off-loading: Practical power management for enterprise storage. ACM Transactions on Storage (TOS) 4, 3 (2008), 10.

[26] Moinuddin K. Qureshi, John Karidis, Michele Franceschini, Vijayalakshmi Srinivasan, Luis Lastras, and Bulent Abali. 2009. Enhancing lifetime and security of PCM-based main memory with start-gap wear leveling. In Proceedings of the 42nd Annual IEEE/ACM International Symposium on Microarchitecture. ACM, 14-23. 
[27] M. J. Rozenberg, I. H. Inoue, and M. J. Sanchez. 2004. Nonvolatile memory with multilevel switching: A basic model. Physical Review Letters 92, 17 (2004), 178302.

[28] Ali Sheikholeslami and P. Glenn Gulak. 2000. A survey of circuit innovations in ferroelectric random-access memories. Proceedings of the IEEE 88, 5 (2000), 667-689.

[29] Shivaram Venkataraman, Niraj Tolia, Parthasarathy Ranganathan, and Roy H. Campbell. 2011. Consistent and durable data structures for non-volatile byte-addressable memory. In FAST, Vol. 11. 61-75.

[30] Guan Wang, Fei Peng, Lei Ju, Lei Zhang, and Zhiping Jia. 2014. Double circulation wear leveling for PCM-based embedded systems. In Advanced Computer Architecture. Springer, 190-200.

[31] H.-S. Philip Wong, Simone Raoux, SangBum Kim, Jiale Liang, John P. Reifenberg, Bipin Rajendran, Mehdi Asheghi, and Kenneth E. Goodson. 2010. Phase change memory. Proceedings of the IEEE 98, 12 (2010), 2201-2227.

[32] Byung-Do Yang, Jae-Eun Lee, Jang-Su Kim, Junghyun Cho, Seung-Yun Lee, and Byoung-Gon Yu. 2007. A low power phase-change random access memory using a data-comparison write scheme. In IEEE International Symposium on Circuits and Systems, 2007 (ISCAS'07). IEEE, 3014-3017.

[33] Joosung Yun, Sunggu Lee, and Sungjoo Yoo. 2012. Bloom filter-based dynamic wear leveling for phase-change RAM. In Proceedings of the Conference on Design, Automation and Test in Europe. EDA Consortium, 1513-1518.

[34] Bo Zhao. 2013. Improving phase change memory (PCM) and spin-torque-transfer magnetic-RAM (STT-MRAM) as next-generation memories: A circuit perspective. Diss. University of Pittsburgh.

[35] Ping Zhou, Bo Zhao, Jun Yang, and Youtao Zhang. 2009. A durable and energy efficient main memory using phase change memory technology. ACM SIGARCH Computer Architecture News 37, 3 (2009), 14-23.

Received August 2017; accepted September 2017 